\title{
Development and Validation of Experimental Modal Analysis with Fixture- Free Oblique Impact Testing Based on Vector Projection Method
}

\author{
Khoo Shin Yee ${ }^{a^{*}}$, Lian Yee Cheng ${ }^{\mathrm{a}}$, Ong Zhi Chao ${ }^{\mathrm{a}}$, Zubaidah Ismail ${ }^{\mathrm{b}}$, Siamak \\ Noroozic \\ ${ }^{a}$ Department of Mechanical Engineering, Engineering Faculty, University of Malaya, 50603 \\ Kuala Lumpur, Malaysia \\ ${ }^{b}$ Department of Civil Engineering, Engineering Faculty, University of Malaya, 50603 Kuala \\ Lumpur, Malaysia
}

${ }^{c}$ School of Design, Engineering \& Computing, Bournemouth University, Poole, Dorset, BH12 $5 B B, U K$

*Corresponding author. Phone number: +603-79677022 (Ext: 2176). Fax number: +6(03)7967 5317. Email: mikeson.khoo@yahoo.com; khooshinyee@um.edu.my

\begin{abstract}
Experimental modal analysis (EMA) with oblique excitation (i.e. oblique impact testing) is useful in improving the long testing time problem of conventional EMA with normal excitation (i.e. tri-axial normal impact testing), in order to extract all important dynamic characteristics of a 3D complex structure. In this study, a new methodology involving vector projection method is introduced to find the driving point frequency response function (FRF) in the oblique direction, without the need of special fixture with oblique-oriented impedance head. Hence, it presents a low cost and practical solution to scale the mode shape, as compared to the traditional *Corresponding author. Tel: +603-79677022 (Ext: 2176); Fax: +6(03)7967 5317

Email: mikeson.khoo@yahoo.com; khooshinyee@um.edu.my
\end{abstract}


approach. Moreover, the concurrent forces characteristic of the oblique excitation is used in the development of the theoretical relationship between the FRF with oblique excitation and normal excitation. This is important for the validation of the oblique impact testing result, such as the FRF and modal parameter estimations. Experimental results show that the oblique impact testing has reliable and effective results, as compared with the tri-axial normal impact testing in terms of the FRF correlation, natural frequency discrepancy, modal damping ratio error and modal assurance criterion (MAC) of the unit modal mass (UMM) mode shape.

Keywords: Concurrent Forces Effect; Oblique Frequency Response Function; Oblique Impact Testing; Oblique Driving Point Measurement; Tri-axial Normal Impact Testing; Vector Projection Method.

*Corresponding author. Tel: +603-79677022 (Ext: 2176); Fax: +6(03)7967 5317

Email: mikeson.khoo@yahoo.com; khooshinyee@um.edu.my 


\section{Introduction}

Any mechanical structure will exhibit vibration when the structure is excited by dynamic forces. Undesirable vibration presents a major hazard and design limitation, especially when excitation matches the structural natural frequency, which greatly amplifies the vibration under the resonance phenomenon. Hence, the identification of structural dynamic properties is important in allowing engineers to prevent the resonance issue [1]. Frequency response function (FRF) testing and modal analysis (MA) are commonly used to identify the dynamic characteristics of a structure. These analyses were introduced several decades ago and have been applied in a wide range of engineering applications such as: validation and updating of finite element model [2, 3], crack detection [4], damage identification [5], force identification [6], linear [7, 8] and nonlinear [9] material property identification, vibration control and structural dynamic design [10]. In general, FRF testing experimentally determines the transfer function that shows the linear relationship between the input force and output response of the system. Then, MA further post-processes the measured transfer function using various curvefitting techniques [11-13] to obtain important dynamic characteristic information with relation to natural frequencies, mode shapes, and modal damping.

Considering a cheaper and more effective solution for small-size structure [14], FRF testing with impact excitation will be implemented in this study. Traditional experimental modal analysis (EMA) requires FRF testing to be conducted in a non-operating condition, where the interruption of operation incurs huge losses. In this scenario, minimizing the testing time of EMA is very crucial. To investigate the dynamic characteristic and dynamic behaviour of a test object, single reference EMA with uni-axial impact/shaker testing is commonly used to provide the solution. However, the uni-axial testing is not suitable for most practical structures whose 
motion is generally complex and multi-directional [15], as it is no longer sufficient to excite all modes of interest from one reference [16]. For example, it is not suitable for the system that will work under a multi-axial excitation environment, such as rotor-induced excitation and aerodynamic loading $[17,18]$, since the uni-axial testing provides only a portion of the complete dynamic characteristic that cannot accurately describe and represent the actual dynamic behaviour of the system under operating condition. To investigate the comprehensive dynamic characteristics of most practical structures, 3 separate uni-axial testing [19] should be performed sequentially at 3 principal directions respectively. However, the sequential threeaxial testing is very time consuming (i.e. increase significantly by a factor of three, as compared to uni-axial testing) due to the redundant procedure [20]. To solve this issue, multi-reference EMA with multi-axial testing such as 3 simultaneous impact/shaker testing in three principal directions [17] can be performed to reduce the testing time and improve the quality of dynamic information. The drawback of this approach is that it requires higher equipment costs and poses difficulty in equipment set-up \& control. The conventional testing above can be further enhanced by using oblique reference EMA with oblique axial impact/shaker testing [21]. This is because a single oblique excitation can induce significant responses in all directions and ensure sufficient participation of all the modes [22]. Thus, the oblique approach saves $2 / 3$ of its total testing time whilst retaining the important dynamic characteristic details in three principal directions.

Oblique reference EMA is firstly introduced and examined in the year 1986, where Døssing [21] designed a special fixture with embedded accelerometer or impedance, to measure the response and excitation in the oblique excitation direction. Shaker and impact testing with oblique reference were conducted and the results were compared with the traditional EMA with uni-axial testing. It showed that the oblique reference exhibited a strong decoupling effect for 
closely spaced mode and thus obtained more accurate modal parameters. Next, Avitabile [23] demonstrated that the oblique reference must be carefully selected at a good location (i.e. antinode) to ensure all the modes of interest were well excited. Besides, the way to obtain the unit modal mass (UMM) mode shape by scaling the residue mode shape using the oblique driving point measurement was also described. Warren et al. [24] implemented the oblique reference EMA with both oblique shaker and oblique impact testing to obtain the FRFs and modal parameters of a base-upright structure. However, the detailed methodology and theoretical validation in applying oblique testing were not provided. Moreover, Baqersad et al. [25] performed the oblique shaker testing to excite both flap-wise and edge-wise modes of a threebladed wind turbine simultaneously. In their experiment, both SIMO and MIMO approaches of the oblique shaker testing failed to excite some modes of the structure, as compared to the sequential two-axial impact testing. This is likely to be due to limitations of the shaker configuration such as the mass loading and rotational inertial loading effects. It is expected that oblique impact testing can produce a better result since it is less affected by those loading effects. However, they did not examine this in their study.

To date, the majority of EMA studies were conducted by using the traditional normal force or perpendicular excitation approach at the desired principal axis. Few studies and publications have been found for the oblique reference EMA especially the oblique impact testing despite its superior testing speed and comprehensive dynamic information. This demonstrates the limitations of the status quo. In many cases, oblique excitation is not desired as it would require a special fixture [26]. This fixture is used mainly for two purposes: (i) To ensure consistency of the impact in the oblique direction; (ii) To make driving point measurements in the oblique direction using a customized impedance head/accelerometer. The need for the special fixture reduces its practicality in terms of implementation (such as raising the concern of mass loading 
and moment effects) as well as increment of equipment cost. On the other hand, the theoretical relationship between the oblique reference EMA and the conventional EMA with normal excitation is yet to be discovered. Thus, many users are still unclear and unconfident to apply the oblique reference EMA, as they prefer to apply the well-established EMA with validated theory. To overcome the drawbacks mentioned above, oblique reference EMA with fixture free will be introduced in this paper, where the driving point measurement is proposed to be carried out by using the vector projection method. Also, this study will establish the theoretical knowledge and relationship between the oblique and normal reference EMAs. The FRF and modal parameters obtained from the proposed oblique reference EMA will be examined and validated with the results of normal reference EMA, to further convince the reliability of the proposed technique.

\section{Theory}

\subsection{Structural FRF Testing with Oblique Impact}

In structural FRF testing, the normal impact procedure is time consuming due to its redundant measurement process. This point is illustrated in Figs. 1(a) - 1(c), where the user needs to repeatedly impact the structure at 3 principal coordinates separately in order to excite all the desired 3D vibration modes. In this paper, the oblique impact will be used to improve the ineffective procedure of the conventional structural FRF testing. A single oblique impact, $F_{o b}$ with pre-determined 3D impact angles (i.e. $\theta_{x}, \theta_{y}$ and $\theta_{z}$ ), is shown in Fig. 1(d), and it can be formulated as an oblique impact vector as follows Eq. (1).

$$
\vec{F}_{o b}=\left|\vec{F}_{o b}\right|\left\{\cos \theta_{x} \hat{\imath}+\cos \theta_{y} \hat{\jmath}+\cos \theta_{z} \hat{k}\right\}
$$

, where $\theta_{x}, \theta_{y}$, and $\theta_{z}$ are the angles between the oblique force and the $x-, y$-, and $z$-axes respectively. $\hat{\imath}, \hat{\jmath}$, and $\hat{k}$ are the unit vectors at $x-, y$-, and $z$-axes respectively. Theoretically, the magnitude of the oblique force, $\left|\vec{F}_{o b}\right|$ or $F_{o b}$ can be transformed to 3 principal coordinates 
using Eq. (2).

$$
\left\{\begin{array}{l}
F_{x} \\
F_{y} \\
F_{z}
\end{array}\right\}=\left|\vec{F}_{o b}\right|\left\{\begin{array}{l}
\cos \theta_{x} \\
\cos \theta_{y} \\
\cos \theta_{z}
\end{array}\right\}
$$

Eq. (3) can be obtained from Eqs. (1) \& (2). It shows that the concurrent normal impacts effect of the oblique impact at 3 principal coordinates. In other words, the oblique impact can represent 3 normal forces (i.e. $F_{x}, F_{y}$, and $F_{z}$ ) acting on the structure at $x$-, $y$-, and $z$-axes simultaneously. With that, the oblique impact could excite all the vibration modes in 3 normal directions simultaneously. Thus, the oblique impact is said to be efficient, to excite 3D vibration modes of a structure, as compared to normal impact.

$$
\vec{F}_{o b}=F_{x} \hat{\imath}+F_{y} \hat{\jmath}+F_{z} \hat{k}
$$

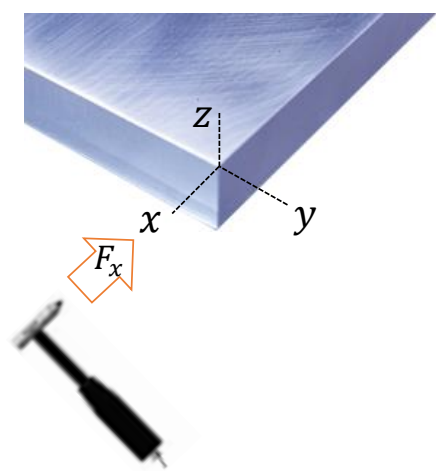

(a)

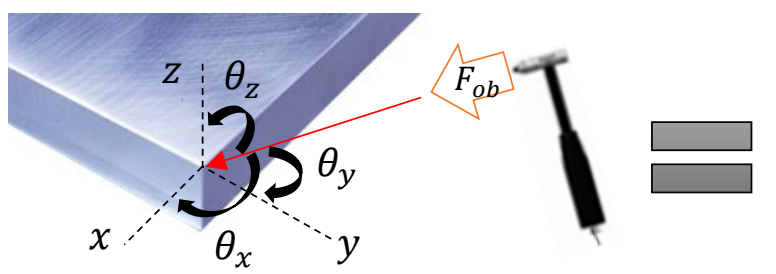<smiles>[Y]C([Y])[Z]</smiles>

(b)
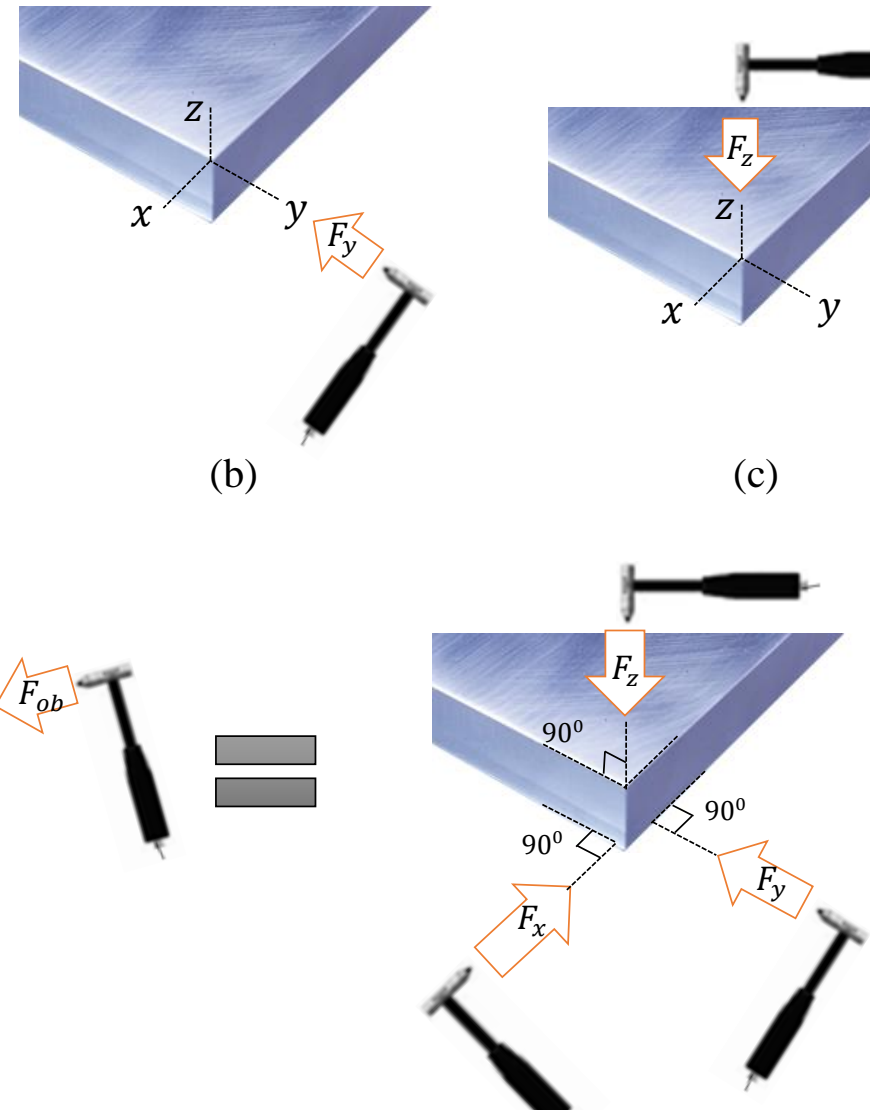

(c)

(d)

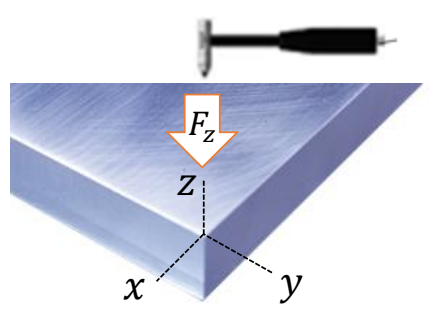

Fig. 1. Structural FRF testing using conventional normal impact at (a) $x-$, (b) $y$-, and (c) $z-$ axes separately, or using (d) oblique impact with concurrent normal impacts effect. 
After obtaining the responses due to the oblique impact at various locations by using a roving tri-axial acceleration, a complete structural FRF can be obtained as follows.

$$
\left[\begin{array}{ccccc}
H_{1 x: 1 o b}(\omega) & H_{1 x: 2 o b}(\omega) & H_{1 x: 3 o b}(\omega) & \cdots & H_{1 x: m j}(\omega) \\
H_{1 y: 1 o b}(\omega) & H_{1 y: 2 o b}(\omega) & H_{1 y: 3 o b}(\omega) & \cdots & H_{1 y: m j}(\omega) \\
H_{1 z: 1 o b}(\omega) & H_{1 z: 2 o b}(\omega) & H_{1 z: 3 o b}(\omega) & \cdots & H_{1 z: m j}(\omega) \\
\vdots & \vdots & \vdots & \ddots & \vdots \\
H_{n i: 1 o b}(\omega) & H_{n i: 2 o b}(\omega) & H_{n i: 3 o b}(\omega) & \cdots & H_{n i: m j}(\omega)
\end{array}\right]=\left[\begin{array}{c}
\ddot{X}_{1 x}(\omega) \\
\ddot{X}_{1 y}(\omega) \\
\ddot{X}_{1 z}(\omega) \\
\vdots \\
\ddot{X}_{n i}(\omega)
\end{array}\right]\left[\begin{array}{c}
F_{1 o b}(\omega) \\
F_{2 o b}(\omega) \\
F_{3 o b}(\omega) \\
\vdots \\
F_{m j}(\omega)
\end{array}\right]^{-1}
$$

, where $H_{n i: m j}$ is the FRF or the transfer function due to input force, $F$ acting at oblique DOF (i.e. location $m$ and direction $j$ ) and its corresponding output acceleration response, $\ddot{X}$ measured at principal coordinates (i.e. location $n$ and direction $i$ ). $[\bullet]^{-1}$ indicates the inversion operation for the matrix. FRF demonstrates the complex input-output relationship in the frequency domain, $\omega$. For a time-invariant linear system, FRF should remain constant as long as the geometric, boundary condition, and material properties of a system are fixed. Moreover, it is not affected by the magnitude and type of the input force.

Instead of measuring a complete FRF matrix (i.e. size $n \times m$ ) as follows Eq. (4), a single column or single row of FRF matrix is sufficient to study the dynamic characteristic or the vibration mode of a structure, as recommended by Richardson and Schwarz [27]. A single column of the FRF matrix (i.e. size $n \times 1$ ) can be obtained through a roving accelerometer approach with a single force DOF reference, while a single row of FRF matrix (i.e. size $1 \times \mathrm{m}$ ) can be obtained using a roving hammer approach with a single response DOF reference [16]. Both approaches can ensure a high quality of FRF measurement as long as the chosen reference DOF is not located at the node point for the vibration modes within the frequency range of interest. By considering these factors, a single-column FRF matrix obtained from the roving accelerometer with single oblique force DOF reference at an anti-node point (e.g. Point \#1) will eventually lead to Eq. (5). 


$$
\left[\begin{array}{c}
H_{1 x: 1 o b}(\omega) \\
H_{1 y: 1 o b}(\omega) \\
H_{1 z: 1 o b}(\omega) \\
\vdots \\
H_{n i: 1 o b}(\omega)
\end{array}\right]=\left[\begin{array}{c}
\ddot{X}_{1 x}(\omega) \\
\ddot{X}_{1 y}(\omega) \\
\ddot{X}_{1 z}(\omega) \\
\vdots \\
\ddot{X}_{n i}(\omega)
\end{array}\right]\left[F_{1 o b}(\omega)\right]^{-1}
$$

Furthermore, driving point FRF measurement must be conducted for mode shape scaling of a dynamic modal model [28]. The driving point FRF can be obtained from the same response and force DOFs in terms of location and direction, e.g. driving point FRF in the oblique direction of Point \#1 is measured as $H_{1 o b: 1 o b}$. Hence, the driving point is included in the FRF measurement with size $((n+1) \times 1)$ as follows Eq. (6).

$$
\left[\begin{array}{c}
H_{1 x: 1 o b}(\omega) \\
H_{1 y: 1 o b}(\omega) \\
H_{1 z: 1 o b}(\omega) \\
\vdots \\
H_{n i: 1 o b}(\omega) \\
H_{1 o b: 1 o b}(\omega)
\end{array}\right]=\left[\begin{array}{c}
\ddot{X}_{1 x}(\omega) \\
\ddot{X}_{1 y}(\omega) \\
\ddot{X}_{1 z}(\omega) \\
\vdots \\
\ddot{X}_{n i}(\omega) \\
\ddot{X}_{1 o b}(\omega)
\end{array}\right]\left[F_{1 o b}(\omega)\right]^{-1}
$$

Traditional impact testing poses no difficulty in measuring the driving point FRF because the tri-axial accelerometer can be easily positioned in the same response direction with the normal impact. Driving point FRF measurement of $H_{1 o b: 1 o b}$ involving oblique impact and tri-axial accelerometer poses measurement difficulty because the orientation of the response sensor does not match or align with the oblique direction. Previous literature [21, 25] implemented special fixture with an additional oblique-oriented impedance head sensor for measuring the force and response in the oblique excitation direction, which made the oblique reference EMA less attractive in practice. To overcome this limitation, the vector projection method is proposed next to find the response in the oblique direction without the use of a special fixture. The oblique response can be measured from the tri-axial responses located in the conventional global coordinate position (i.e. axial, horizontal, and vertical directions of the test object). 
Assuming that the oblique impact direction (i.e. $\theta_{x}, \theta_{y}$, and $\theta_{z}$ ) can be pre-defined prior to any structural frequency response testing, the corresponding oblique response vector in the time domain, $\vec{X}_{1 o b}(t)$ can be found through the vector projection of the resultant tri-axial responses to the oblique direction as follows Eqs. (7) - (10). In addition, the magnitude of the oblique response, $\ddot{X}_{1 o b}(t)$ or $\left|\vec{X}_{1 o b}(t)\right|$ can be computed using the scalar projection, i.e. dot product between the total tri-axial response vector, $\overrightarrow{\ddot{X}}_{1, \text { total }}(t)$ and the unit vector of the oblique impact direction, $\hat{\theta}_{o b}(t)$, as follows Eq. (10). Hence, the frequency-domain oblique response, $\ddot{X}_{1 o b}(\omega)$ can be obtained through a Discrete Fourier Transform (DFT). Together with the measured oblique impact directly from the instrumented impact hammer, the oblique driving point FRF can be obtained using $H_{1 o b: 1 o b}(\omega)=\ddot{X}_{1 o b}(\omega) / F_{1 o b}(\omega)$.

$$
\overrightarrow{\ddot{X}}_{1 o b}(t)=\left\{\overrightarrow{\ddot{X}}_{1, t o t a l}(t) \cdot \hat{\theta}_{o b}(t)\right\} \hat{\theta}_{o b}(t)
$$

, where

$$
\begin{gathered}
\overrightarrow{\ddot{X}}_{1, \text { total }}(t)=\ddot{X}_{1 x}(t) \hat{\imath}+\ddot{X}_{1 y}(t) \hat{\jmath}+\ddot{X}_{1 z}(t) \hat{k} \\
\hat{\theta}_{o b}(t)=\cos \theta_{x}(t) \hat{\imath}+\cos \theta_{y}(t) \hat{\jmath}+\cos \theta_{z}(t) \hat{k} \\
\ddot{X}_{1 o b}(t)=\left|\overrightarrow{\ddot{X}}_{1 o b}(t)\right|=\left\{\overrightarrow{\ddot{X}}_{1, \text { total }}(t) \cdot \hat{\theta}_{o b}(t)\right\}
\end{gathered}
$$

\subsection{Experimental Modal Analysis (EMA) with Oblique Impact}

A general procedure of EMA mainly contains two parts: (i) FRF measurement and (ii) modal parameter estimation. For part (i), a complete column of the FRF matrix with an additional oblique driving point follows Eq. (6) can be successfully obtained using oblique impact testing introduced in Section 2.1. Next, part (ii) - modal parameter estimation from the FRF will be discussed, to extract the dynamic characteristics of a structure. 
Curve fitting the measured FRFs to the modal model is a common approach to estimate the dynamic characteristics of a linear time-invariant system. According to Richardson and Schwarz [27], the experimental FRF matrix obtained from Eq. (6) can be written in partial fraction expansion form, as follows Eq. (11), which is a frequency domain curve fitting model.

$$
\underbrace{\left[\mathbf{H}_{n i: 1 o b}(\omega)\right]}_{(n+1) x 1}=\sum_{k=1}^{m} \frac{\left[\mathbf{r}_{n i: 10 b, k}\right]}{\left(s-\lambda_{k}\right)}-\left.\frac{\left[\mathbf{r}_{n i: 1 o b, k}\right]^{*}}{\left(s-\lambda_{k}^{*}\right)}\right|_{s=j \omega}
$$

, where $j=\sqrt{-1}, \lambda_{k}=-\sigma_{k}+j \omega_{d, k}$ is the pole location of the $k^{\text {th }}$ vibration mode, which consists the decay rate, $\sigma_{k}$ in the real part and damped natural frequency, $\omega_{d, k}$ in the imaginary part, $s$ is the Laplace domain coefficient, $\bullet^{*}$ is the complex conjugate function and $\left[\mathbf{H}_{n i: 1 o b}(\omega)\right]$ is the $(n+1)$ by 1 column measurement of the FRF matrix with an additional row of the oblique driving point information as follows Eq. (6). Note that the modal model is only evaluated along the imaginary axis of the S-plane (i.e. $s=j \omega$ ) because the FRF matrix is measured in the frequency domain. $\left[\mathbf{r}_{n i: 1 o b, k}\right]$ is the $k^{\text {th }}$ mode $(n+1)$ by 1 residue mode shape matrix. It can be scaled to UMM mode shape by using Eq. (12).

$$
\left[\boldsymbol{\varphi}_{n i, k}\right]=\frac{1}{A_{k} \varphi_{1 o b, k}}\left[\mathbf{r}_{n i: 1 o b, k}\right]
$$

, where the oblique driving point measurement will eventually produce UMM scaling factor of the $k^{t h}$ vibration mode, $A_{k}=r_{1 o b: 1 o b, k} /\left(\varphi_{1 o b, k}\right)^{2}$. On the other hand, the scaling factor, $A_{k}=$ $1 / \omega_{d, k}$ can be obtained according to the UMM property [29] by setting the modal mass equal to one. With that, Eq. (12) can be further simplified to Eq. (13), which can be used to determine the UMM mode shape from the residue mode shape.

$$
\left\{\begin{array}{c}
\varphi_{1 x, k} \\
\varphi_{1 y, k} \\
\varphi_{1 z, k} \\
\vdots \\
\varphi_{n i, k} \\
\varphi_{1 o b, k}
\end{array}\right\}=\sqrt{\frac{\omega_{d, k}}{r_{1 o b: 1 o b, k}}}\left\{\begin{array}{c}
r_{1 x: 1 o b, k} \\
r_{1 y: 1 o b, k} \\
r_{1 z: 1 o b, k} \\
\vdots \\
r_{n i: 1 o b, k} \\
r_{1 o b: 1 o b, k}
\end{array}\right\}
$$




\subsection{Validation of the FRF Result Obtained from the Oblique Impact Testing}

As shown in Eq. (3), an oblique impact can excite a structure at 3 principal coordinates, hence makes possible the simplification of the redundancy testing procedure for the conventional, normal impacts approach. In fact, an oblique impact can be represented by three concurrent normal impacts acting on a structure as shown in Fig. 1(d). To access the FRF measurement and modal parameters' estimation qualities of the proposed oblique impact testing, result validation with the conventional tri-axial normal impact testing result will be discussed next.

Firstly, the normal FRF testing will be conducted by using 3 distinct normal impacts (i.e. $F_{x}$, $F_{y}$, and $F_{z}$ acting at 3 principal coordinates separately) and roving accelerometer at $n$ locations. In general, a 3D structure requires minimum of 3 rows or columns to comprehensively define the dynamic characteristics from each direction. This indicates that the conventional data acquisition process has to be repeated 3 times when conducting a normal FRF testing. Moreover, the reference force DOF must be located at an anti-node point (e.g. Point \#1). By considering the factors above, the FRF matrix obtained through tri-axial normal impact testing at 3 principal coordinates separately is shown in Eq. (14).

$$
\left[\begin{array}{ccc}
H_{1 x: 1 x}(\omega) & H_{1 x: 1 y}(\omega) & H_{1 x: 1 z}(\omega) \\
H_{1 y: 1 x}(\omega) & H_{1 y: 1 y}(\omega) & H_{1 y: 1 z}(\omega) \\
H_{1 z: 1 x}(\omega) & H_{1 z: 1 y}(\omega) & H_{1 z: 1 z}(\omega) \\
\vdots & \vdots & \vdots \\
H_{n i: 1 x}(\omega) & H_{n i: 1 y}(\omega) & H_{n i: 1 z}(\omega)
\end{array}\right]=\left[\begin{array}{c}
\ddot{X}_{1 x}(\omega) \\
\ddot{X}_{1 y}(\omega) \\
\ddot{X}_{1 z}(\omega) \\
\vdots \\
\ddot{X}_{n i}(\omega)
\end{array}\right]\left[\begin{array}{c}
F_{1 x}(\omega) \\
F_{1 y}(\omega) \\
F_{1 z}(\omega)
\end{array}\right]^{-1}
$$

For the concurrent normal impacts acting on the structure in 3 principal directions simultaneously, the resultant response due to the superposition of vibration modes can be obtained from Eq. (15). 


$$
\left[\begin{array}{c}
\ddot{X}_{1 x}(\omega) \\
\ddot{X}_{1 y}(\omega) \\
\ddot{X}_{1 z}(\omega) \\
\vdots \\
\ddot{X}_{n i}(\omega)
\end{array}\right]=\left[\begin{array}{c}
H_{1 x: 1 x}(\omega) F_{1 x}(\omega)+H_{1 x: 1 y}(\omega) F_{1 y}(\omega)+H_{1 x: 1 z}(\omega) F_{1 z}(\omega) \\
H_{1 y: 1 x}(\omega) F_{1 x}(\omega)+H_{1 y: 1 y}(\omega) F_{1 y}(\omega)+H_{1 y: 1 z}(\omega) F_{1 z}(\omega) \\
H_{1 z: 1 x}(\omega) F_{1 x}(\omega)+H_{1 z: 1 y}(\omega) F_{1 y}(\omega)+H_{1 z: 1 z}(\omega) F_{1 z}(\omega) \\
\vdots \\
H_{n i: 1 x}(\omega) F_{1 x}(\omega)+H_{n i: 1 y}(\omega) F_{1 y}(\omega)+H_{n i: 1 z}(\omega) F_{1 z}(\omega)
\end{array}\right]
$$

Since the oblique impact has the concurrent normal impacts effect, Eq. (2) can be substituted to Eq. (15) and rearrange it to form Eq. (16).

$$
\left[\begin{array}{c}
H_{1 x: 1 x} \cos \theta_{x}+H_{1 x: 1 y} \cos \theta_{y}+H_{1 x: 1 z} \cos \theta_{z} \\
H_{1 y: 1 x} \cos \theta_{x}+H_{1 y: 1 y} \cos \theta_{y}+H_{1 y: 1 z} \cos \theta_{z} \\
H_{1 z: 1 x} \cos \theta_{x}+H_{1 z: 1 y} \cos \theta_{y}+H_{1 z: 1 z} \cos \theta_{z} \\
\vdots \\
H_{n i: 1 x} \cos \theta_{x}+H_{n i: 1 y} \cos \theta_{y}+H_{n i: 1 z} \cos \theta_{z}
\end{array}\right]=\left[\begin{array}{c}
\ddot{X}_{1 x}(\omega) \\
\ddot{X}_{1 y}(\omega) \\
\ddot{X}_{1 z}(\omega) \\
\vdots \\
\ddot{X}_{n i}(\omega)
\end{array}\right]\left[F_{1 o b}(\omega)\right]^{-1}
$$

Eq. (16) can be further simplified as following: $\left[\mathbf{H}_{n i: 1 x y z}\right]=\left[\mathbf{H}_{n i: 1 x}\right] \cos \theta_{x}+$ $\left[\mathbf{H}_{n i: 1 y}\right] \cos \theta_{y}+\left[\mathbf{H}_{n i: 1 z}\right] \cos \theta_{z}$, which represents the FRFs matrix due to 3 concurrent normal impacts acting at Point \#1 and various responses at ni DOF. It is worthwhile to mention that the FRFs will change depending on the oblique impact direction. For example, if the oblique impact is close to the $z$-direction (where $\theta_{z} \approx 0^{0}$ and $\theta_{x}=\theta_{y} \approx 90^{\circ}$ ), then the FRF due to the concurrent impacts can be reduced to FRF due to $Z$-axis normal impact, i.e. $\left[\mathbf{H}_{n i: 1 x y z}\right] \approx$ $\left[\mathbf{H}_{n i: 1 z}\right]$. Thus, the impact direction, $\hat{\theta}_{o b}$ should be selected carefully so that it can adequately excite the vibration modes at 3 principal coordinates.

In fact, $\left[\mathbf{H}_{n i: 1 x}\right],\left[\mathbf{H}_{n i: 1 y}\right]$, and $\left[\mathbf{H}_{n i: 1 z}\right]$ can be obtained from the conventional FRF testing with 3 distinct normal impacts at $x-, y-$, and $z$-axes respectively (i.e. tri-axial normal impact testing). By comparing the Eqs. (5) and (16), the relationship between the oblique FRF and the normal FRF obtained from the oblique impact testing and tri-axial normal impact testing respectively, is established, where the oblique FRF, $\left[\mathbf{H}_{n i: 1 o b}\right]$ is equal to the superpositionnormal FRF, $\left[\mathbf{H}_{n i: 1 x y z}\right]$ theoretically. This theoretical relationship will be validated experimentally in this study. Once the impact direction, $\hat{\theta}_{o b}$ is measured in a priori, the FRF 
result acquired from the experimental oblique impact testing, i.e. $\left[\mathbf{H}_{n i: 1 o b}\right]$ in Eq. (5) can be validated with the FRF result acquired from the experimental tri-axial normal impact testing $\left[\mathbf{H}_{n i: 1 x y z}\right]$ in Eq. (16).

In addition, the driving point FRF due to 3 concurrent normal impacts acting at Point \#1 can be obtained using $H_{1 x y z: 1 x y z}(\omega)=\ddot{X}_{1 x y z}(\omega) / F_{1 x y z}(\omega)$, where $\ddot{X}_{1 x y z}(\omega)$ and $F_{1 x y z}(\omega)$ are the total response and total concurrent impact force at oblique direction of Point \#1 respectively. They can be obtained through the DFT and vector projection method, similar to Eqs. (7)-(10) in Section 2.1 to complete the Eq. (17). For the sake of brevity, the vector projection equations to obtain the $\ddot{X}_{1 x y z}(\omega)$ and $F_{1 x y z}(\omega)$ are not provided here. Note that this driving point FRF will be useful for the mode shape scaling purpose later.

$$
\left[\begin{array}{c}
H_{1 x: 1 x} \cos \theta_{x}+H_{1 x: 1 y} \cos \theta_{y}+H_{1 x: 1 z} \cos \theta_{z} \\
H_{1 y: 1 x} \cos \theta_{x}+H_{1 y: 1 y} \cos \theta_{y}+H_{1 y: 1 z} \cos \theta_{z} \\
H_{1 z: 1 x} \cos \theta_{x}+H_{1 z: 1 y} \cos \theta_{y}+H_{1 z: 1 z} \cos \theta_{z} \\
\vdots \\
H_{n i: 1 x} \cos \theta_{x}+H_{n i: 1 y} \cos \theta_{y}+H_{n i: 1 z} \cos \theta_{z} \\
H_{1 x y z: 1 x y z}
\end{array}\right]=\left[\begin{array}{c}
\ddot{X}_{1 x}(\omega) \\
\ddot{X}_{1 y}(\omega) \\
\ddot{X}_{1 z}(\omega) \\
\vdots \\
\ddot{X}_{n i}(\omega) \\
\ddot{X}_{1 o b}(\omega)
\end{array}\right]\left[F_{1 o b}(\omega)\right]^{-1}
$$

\subsection{Validation of the Modal Parameters' Results Obtained from the Oblique Impact Testing}

Modal parameters obtained from the oblique impact testing, such as the damped natural frequency, modal damping ratio, and UMM mode shape will be validated with the conventional tri-axial normal impact testing result as follows. Similar to the previous case, the superpositionnormal FRF will be fitted to the model in Eq. (18) for modal parameters estimation.

$$
\underbrace{\left[\mathbf{H}_{n i: 1 x y z}(\omega)\right]}_{(n+1) x 1}=\sum_{k=1}^{m} \frac{\left[\mathbf{r}_{n i: 1 x y z, k}\right]}{\left(s-\lambda_{k}\right)}-\left.\frac{\left[\mathbf{r}_{n i: 1 x y z, k}\right]^{*}}{\left(s-\lambda_{k}^{*}\right)}\right|_{s=j \omega}
$$

, where the pole location consists of the damped natural frequency and modal damping ratio information at each vibration mode. Note that $\left[\mathbf{H}_{n i: 1 x y z}(\omega)\right]$ is the $(n+1)$ by 1 FRF matrix 
obtained from Eq. (17), with an additional row for the driving point information. $\left[\mathbf{r}_{n i: 1 x y z, k}\right]$ is the $k^{\text {th }}$ mode $(n+1)$ by 1 residue mode shape matrix and it can be scaled to UMM mode shape by using Eq. (19). Then, the extracted modal parameters from the oblique FRF using Eqs. (11) and (13) will be compared with extracted modal parameters from the superpositionnormal FRF using Eqs. (18) and (19). Hence, the accuracy of oblique impact testing in modal parameter estimation can be validated.

$$
\left\{\begin{array}{c}
\varphi_{1 x, k} \\
\varphi_{1 y, k} \\
\varphi_{1 z, k} \\
\vdots \\
\varphi_{n i, k} \\
\varphi_{1 x y z, k}
\end{array}\right\}=\sqrt{\frac{\omega_{d, k}}{r_{1 x y z: 1 x y z, k}}}\left\{\begin{array}{c}
r_{1 x: 1 x y z, k} \\
r_{1 y: 1 x y z, k} \\
r_{1 z: 1 x y z, k} \\
\vdots \\
r_{n i: 1 x y z, k} \\
r_{1 x y z: 1 x y z, k}
\end{array}\right\}
$$

\section{Material and Method}

\subsection{Experimental Setup}

In this study, a T-shaped aluminium plate consisting of a motor coupled to a rotor shaft is used as a test rig. The motor's maximum operating frequency is $50 \mathrm{~Hz}$ and it is in shutdown condition during the FRF testing. A total of 19 measurement points are set across the plate, as shown in Fig. 2. A tri-axial accelerometer (model PCB® 356B18) with sensitivity $1000 \mathrm{mV} / \mathrm{g}$ is used to measure the corresponding translational accelerations at 3 principal directions of the plate, as illustrated in Fig. 2. The measurement frequency range $( \pm 10 \%)$ of the accelerometer is $0.5-5000 \mathrm{~Hz}$, which is sufficient to measure the vibration due to impact. A modally tuned impact hammer (model Dytran® 5800B30) with sensitivity $10.91 \mathrm{mV} / \mathrm{N}$ is used to excite the test rig. The allowable amplitude range of the impact hammer is $444.8 \mathrm{~N}$. The impact hammer with a rubber tip is chosen as it can excite the vibration modes of interest up to $100 \mathrm{~Hz}$. 


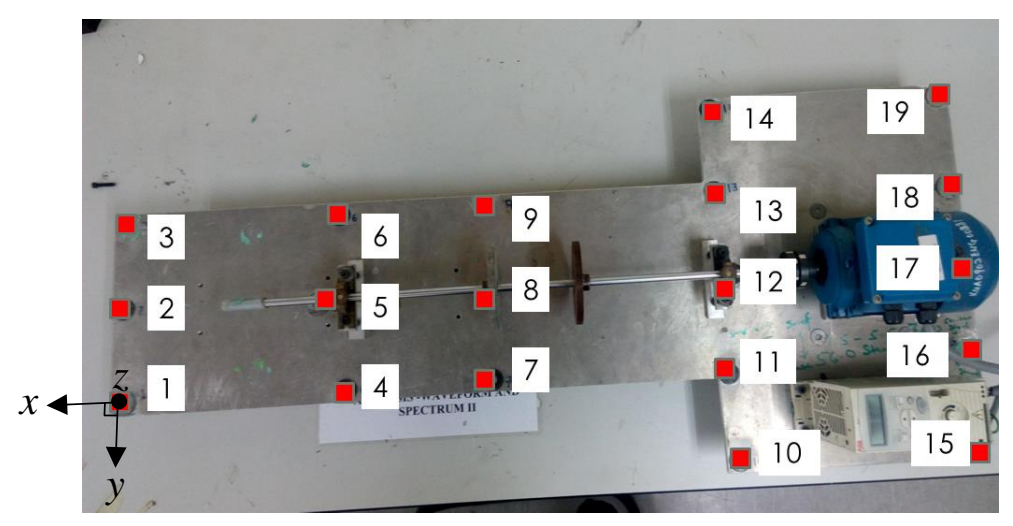

Fig. 2. T-shaped aluminium plate.

Both force and response signals are acquired by a 4-channel data acquisition system (DAQ model NIß 9234) and the raw data is collected using an in-house measurement system with a virtual instrument software (DASYLAB®). The block size and sampling rate of the FRF measurement are set at 4096 samples and $2048 \mathrm{~Hz}$ respectively. This setting gives a time resolution of $0.0004882 \mathrm{~s}$ and frequency resolution of $0.5 \mathrm{~Hz}$, which are sufficient to measure both impact force and response signals satisfactorily. The measurement time of each block is around 2 s. Once the impact force exceeds the threshold value (i.e. $10 \mathrm{~N}$ ) of a trigger function, both response and force signals will be measured with a pre-trigger of 50 samples. Besides, the post-trigger of the remaining 4046 is selected. A total of 50 averages for each measurement are selected to reduce the measurement noise. Zero ends are observed in the response and force signals, where the entire signals are captured within the sample interval. Therefore, a rectangular window with a magnitude of one is selected before applying the fast Fourier transform (FFT) function. No spectrum leakage is observed in the FFT result indicates the selection of windowing function is appropriate. As follows ISO 7626-1:2011 [30], the FFT results of both response and force signals due to transient vibration are used to determine the FRF result as following: $H_{n i: m j}(\omega)=\ddot{X}_{n i}(\omega) / F_{m j}(\omega)$, where $n$ and $m$ are the position DOFs, while $i$ and $j$ are the direction DOFs, for the response and force signals respectively. $\omega$ indicates that the operation is in the frequency domain. With that, the setting of the FRF measurement is ready. 
Next, the post-processing of the vibration data will be performed. Firstly, the calculation of vector projection is performed in a numerical computing software (Matlab®) to project the triaxial response or tri-axial normal force signals into the oblique direction. Hence, driving point FRF in the oblique direction can be obtained. Once all FRF data including the driving point FRF is ready, ME'scope ${ }^{\circledR}$ will be used to curve fit the FRF for modal parameter extraction purpose. In this study, a polynomial curve fitting algorithm is used to obtain the natural frequencies, modal damping ratio, and residue mode shapes. The residue mode shapes are then scaled to UMM mode shapes by using the scaling function in ME'scope ${ }^{\circ}$. A 3D wire-linked structural model of the T-plate test rig can be drawn in ME'scope ${ }^{\circledR}$ so that the animation of mode shape can be performed. With that, the setting of the oblique driving point FRF and modal parameter estimation is ready.

\subsection{Experimental Procedure for Oblique Impact Testing}

The single input single output (SISO) approach is adopted for the FRF measurement with oblique excitation. The current research scope focuses on a single oblique direction. The oblique reference point (i.e. fixed oblique impact) is set at Point \#1 with oblique angle $\left\{\theta_{x}, \theta_{y}, \theta_{z}\right\}$ of $\left\{60^{\circ}, 60^{\circ}, 45^{\circ}\right\}$, by using global coordinate defined in Figs. 1 and 2 . In this study, supporting component and protractor tools are used as a guide rail for oblique impact as shown in Fig. 3.

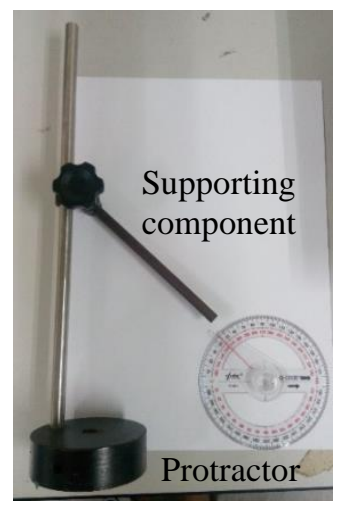

Fig. 3. Supporting component for oblique impact. 
Similar to the traditional normal impact testing approach, the user is required to maintain the oblique impact at the pre-determined location and direction. The steps to ensure consistent oblique impact are given below:

(i) Perform the polar-to-spherical coordinate transformation. For example, $\left\{\theta_{x}, \theta_{y}, \theta_{z}\right\}$ in polar coordinate can be converted to $\left\{\theta_{a}, \theta_{p}\right\}$ in spherical coordinate, where $\theta_{a}=$ $\tan ^{-1}\left(\cos \theta_{y} / \cos \theta_{x}\right)$ and $\theta_{p}=\theta_{z}$ are the azimuthal and polar angles respectively. Hence, $\left\{\theta_{a}, \theta_{p}\right\}=\left\{45^{0}, 45^{0}\right\}$ can be obtained from $\left\{\theta_{x}, \theta_{y}, \theta_{z}\right\}=\left\{60^{\circ}, 60^{\circ}, 45^{\circ}\right\}$.

(ii) Adjust the supporting component to the desired impact direction according to the spherical coordinate as illustrated in Fig. 4. A protractor tool is used to measure the angle of the supporting component. The supporting component is initially pointing at the $x$-axis of Point \#1, then rotates $45^{\circ}$ towards positive y-axis (i.e. $\theta_{a}$ in Fig. $4(\mathrm{~b})$ ). Lastly, rotate it $45^{\circ}$ towards positive $z$-axis (i.e. $90^{\circ}-\theta_{p}$ in Fig. $4($ c)) .

(iii) Additional supporting component \#2 is added parallel to the supporting component \#1, in order to form the guide rail for the oblique impact as shown in Fig. 5. In this way, impact in the oblique direction can be held within acceptable limits as follows ISO 7626-5:1994 [31]. Hence, consistent oblique impact can be achieved. 

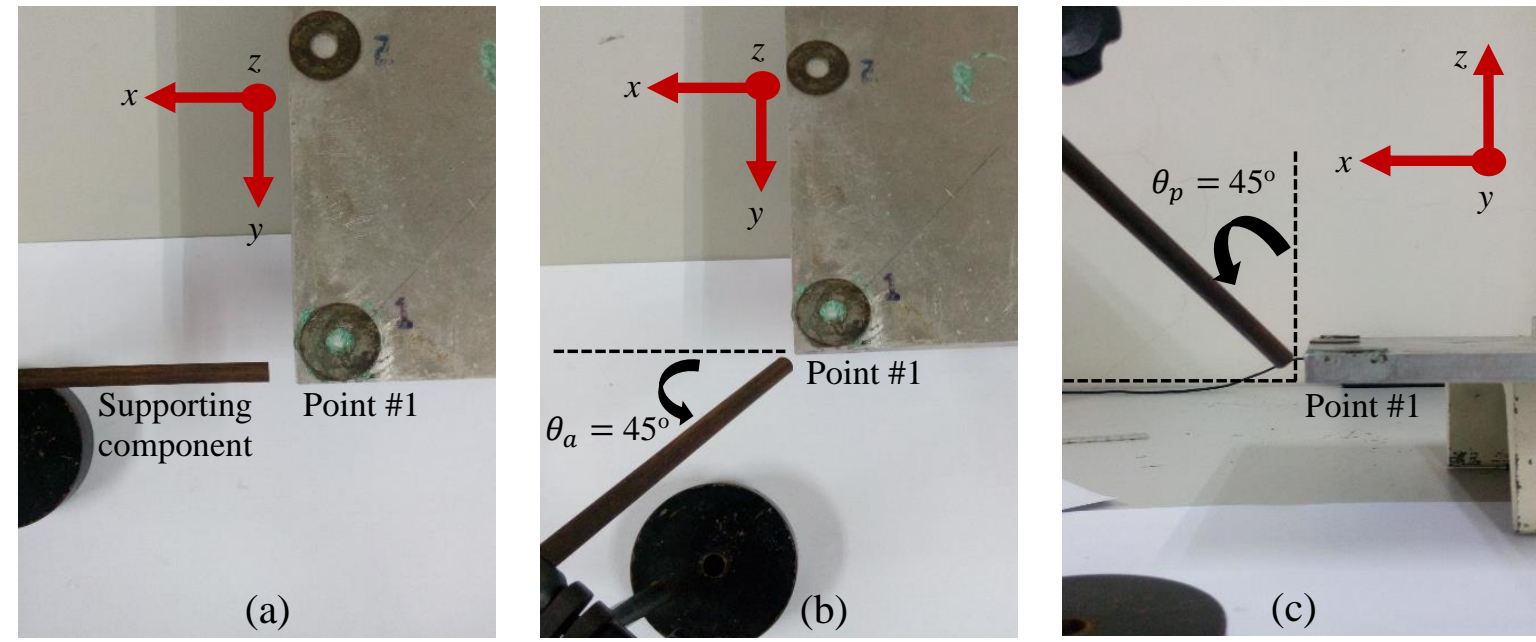

Fig. 4. The positioning of the supporting component to the pre-determined oblique direction.

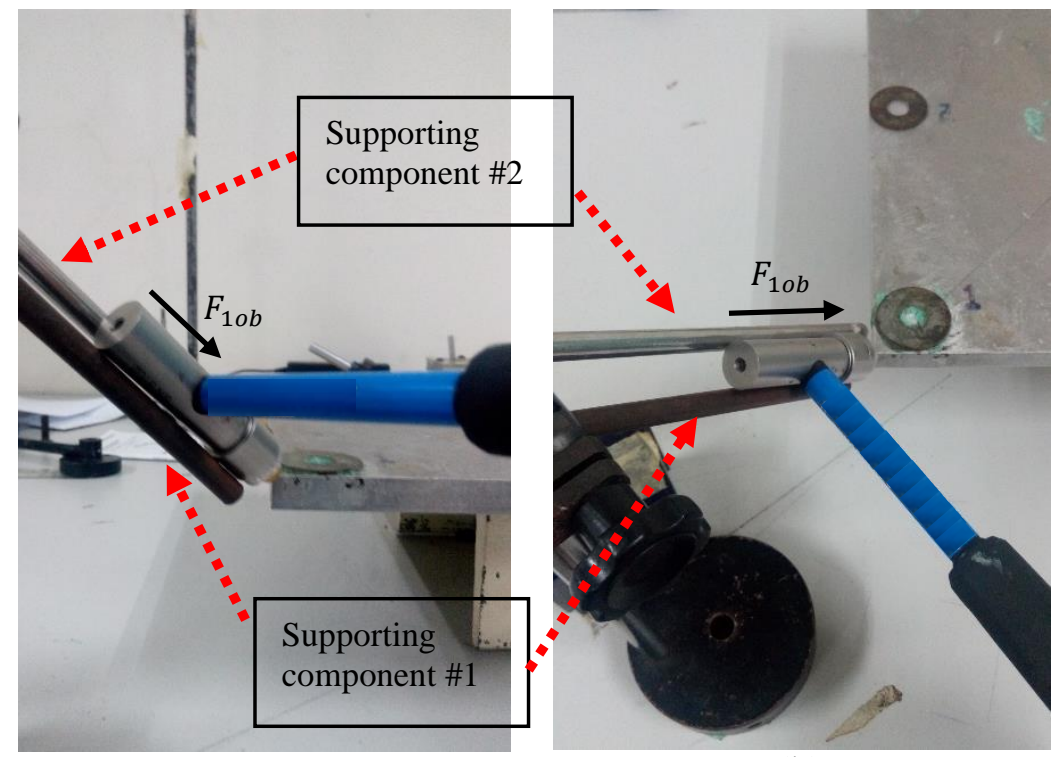

(a)

(b)

Fig. 5. (a) Side and (b) isometric views of the oblique impact with the guide rail.

Next, the tri-axial accelerometer is roved from Points \#1 to \#19 to record the corresponding impact-induced response at the $x$-, $y$-, and $z$-axes. A total of 50 averages are used for each measurement. Thus, in total, 57 FRFs are collected at 19 measurement points and the data acquisition procedure requires 950 impacts. In Matlab®, the response in the direction of the oblique impulse can be calculated through the proposed vector projection method by using Eqs. (7) - (10). Hence, the additional oblique driving point FRF can be obtained. All the measured 
data $(57+1)$ by 1 FRF matrix can be arranged as follows Eq. (6) and it will be further postprocessed to obtain the dynamic characteristics such as damped natural frequencies, residue mode shape, and UMM mode shape using ME'scope ${ }^{\circledR}$. Next, the accuracy of the oblique impact testing result will be benchmarked with the tri-axial normal impact testing result.

\subsection{Experimental Procedure for Tri-Axial Normal Impact Testing}

Conventional tri-axial normal impact testing is carried out to validate the result of oblique impact testing. SISO roving accelerometer approach is adopted, where the Point \#1 is set as the reference point for the normal force (i.e. fixed normal impact). Firstly, the test rig is impacted at Point $\# 1$ in the $x$-direction (i.e. $\left\{\theta_{x}, \theta_{y}, \theta_{z}\right\}=\left\{0^{\circ}, 90^{\circ}, 90^{\circ}\right\}$ ), as shown in Fig. 6(a) while the tri-axial accelerometer is roving from Points \#1 to 19 for 19 measurements. A total of 50 averages are used for each measurement. This procedure is repeated for the impact in the y- and $z$-directions respectively (i.e. $\left\{90^{\circ}, 0^{\circ}, 90^{\circ}\right\} \&\left\{90^{\circ}, 90^{\circ}, 0^{\circ}\right\}$ ) as shown in Figs. 6(b) and (c). A total of 57 FRFs are collected for each normal impact, at 19 measurement points and the data acquisition procedure requires 2850 impacts (i.e. 950 impacts $x 3$ directions). Therefore, the FRF matrix with size (57 x 3) can be obtained as follows Eq. (14).
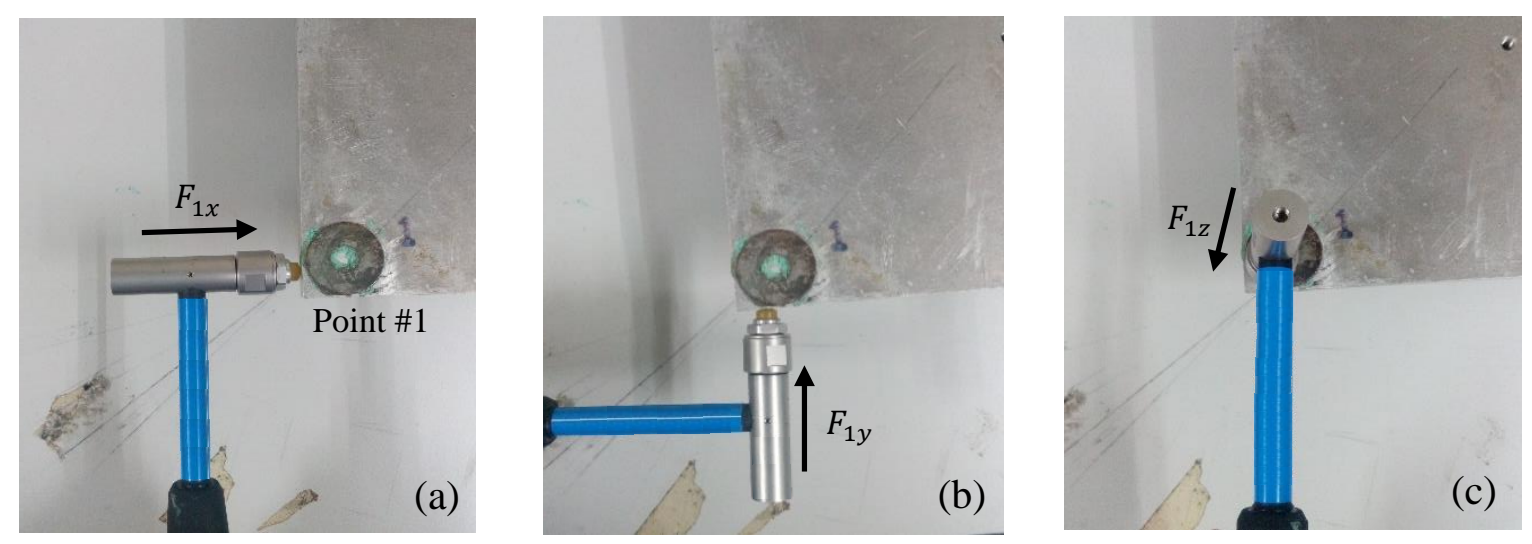

Fig. 6. Normal impact testing in (a) $x$-, (b) $y$-, and (c) $z$-directions. 


\subsection{Validation Method for FRF Results Obtained from Oblique Impact Testing}

Theoretically, FRF results of oblique impact testing have the same effects as the tri-axial normal impact testing with concurrent forces, as discussed in Section 2. To validate this point, the measured FRFs due to the oblique impact from Eq. (5) will be compared with the measured FRFs due to 3 concurrent normal impacts from Eq. (16), as illustrated in Eq. (20). Note that the validity of Eq. (20) will greatly demonstrate the time effective oblique impact testing can be performed instead of the time-consuming normal impact testing.

$$
\left[\begin{array}{c}
H_{1 x: 1 o b}(\omega) \\
H_{1 y: 1 o b}(\omega) \\
H_{1 z: 1 o b}(\omega) \\
\vdots \\
H_{19 x: 1 o b}(\omega) \\
H_{19 y: 1 o b}(\omega) \\
H_{19 z: 1 o b}(\omega)
\end{array}\right]=\left[\begin{array}{c}
H_{1 x: 1 x} \cos \theta_{x}+H_{1 x: 1 y} \cos \theta_{y}+H_{1 x: 1 z} \cos \theta_{z} \\
H_{1 y: 1 x} \cos \theta_{x}+H_{1 y: 1 y} \cos \theta_{y}+H_{1 y: 1 z} \cos \theta_{z} \\
H_{1 z: 1 x} \cos \theta_{x}+H_{1 z: 1 y} \cos \theta_{y}+H_{1 z: 1 z} \cos \theta_{z} \\
\vdots \\
H_{19 x: 1 x} \cos \theta_{x}+H_{19 x: 1 y} \cos \theta_{y}+H_{19 x: 1 z} \cos \theta_{z} \\
H_{19 y: 1 x} \cos \theta_{x}+H_{19 y: 1 y} \cos \theta_{y}+H_{19 y: 1 z} \cos \theta_{z} \\
H_{19 z: 1 x} \cos \theta_{x}+H_{19 z: 1 y} \cos \theta_{y}+H_{19 z: 1 z} \cos \theta_{z}
\end{array}\right]
$$

In this study, the oblique direction is set at $\left\{60^{\circ}, 60^{\circ}, 45^{\circ}\right\}$. Hence, Eq. (20) can be simplified to Eq. (21). It shows that the oblique FRF is due to the superposition of normal reference FRFs in three principal directions, which illustrates the 'concurrent forces effect'.

$$
\left[\begin{array}{c}
H_{1 x: 1 o b}(\omega) \\
H_{1 y: 1 o b}(\omega) \\
H_{1 z: 1 o b}(\omega) \\
\vdots \\
H_{19 x: 1 o b}(\omega) \\
H_{19 y: 1 o b}(\omega) \\
H_{19 z: 1 o b}(\omega)
\end{array}\right]=0.5\left[\begin{array}{c}
H_{1 x: 1 x}(\omega) \\
H_{1 y: 1 x}(\omega) \\
H_{1 z: 1 x}(\omega) \\
\vdots \\
H_{19 x: 1 x}(\omega) \\
H_{19 y: 1 x}(\omega) \\
H_{19 z: 1 x}(\omega)
\end{array}\right]+0.5\left[\begin{array}{c}
H_{1 x: 1 y}(\omega) \\
H_{1 y: 1 y}(\omega) \\
H_{1 z: 1 y}(\omega) \\
\vdots \\
H_{19 x: 1 y}(\omega) \\
H_{19 y: 1 y}(\omega) \\
H_{19 z: 1 y}(\omega)
\end{array}\right]+0.707\left[\begin{array}{c}
H_{1 x: 1 z}(\omega) \\
H_{1 y: 1 z}(\omega) \\
H_{1 z: 1 z}(\omega) \\
\vdots \\
H_{19 x: 1 z}(\omega) \\
H_{19 y: 1 z}(\omega) \\
H_{19 z: 1 z}(\omega)
\end{array}\right]
$$

If Eq. (21) is true, then the 'concurrent forces effect' of the oblique FRF can be validated. Validation of oblique FRF is conducted against the superposition-normal FRF, via the comparison of peak location, peak amplitude, and the number of peaks. Further validation is conducted by investigating the correlation between the oblique FRF and superposition-normal FRF. A total of 57 oblique FRFs from oblique impact testing and 57 superposition-normal 
FRFs from tri-axial normal impact testing at three axes are averaged respectively. Correlation, Corr between both mean FRFs are inspected using Eq. (22).

$$
\operatorname{Corr}=\frac{\left|\sum_{r=0}^{B S-1}\left(O_{r} S_{r}^{*}\right)\right|^{2}}{\left(\sum_{r=0}^{B S-1}\left(O_{r} O_{r}^{*}\right)\right)\left(\sum_{r=0}^{B S-1}\left(S_{r} S_{r}^{*}\right)\right)}
$$

, where $O_{r}$ and $S_{r}$ are the $r^{\text {th }}$ samples of the mean oblique FRF and the mean superpositionnormal FRF respectively. $\bullet^{*}$ is the complex conjugate function. Block size, $B S$ is the total number of collected samples per block. Note that the correlation value greater than 0.9 indicates an excellent agreement between the measured oblique FRF and the conventional superpositionnormal FRFs.

\subsection{Validation Method for Modal Parameters Result Obtained from Oblique Impact Testing}

By using ME'scope ${ }^{\circledR}$ software, the measured oblique FRFs and superposition-normal FRFs are curve-fitted separately to extract its corresponding natural frequency, modal damping ratio, and residue mode shapes. The deviation between the natural frequency, and modal damping ratio will be compared for both tests. As a rule of thumb, natural frequency and modal damping ratio errors within $5 \%$ deviation is considered good and acceptable. To obtain UMM mode shape, additional driving point FRF in the oblique direction is required for the oblique and triaxial normal impact testing respectively, as follows Eqs. (6) and (17). Hence, the mode shape animation by these two tests will be compared side by side. Moreover, the mode shape comparison can be done using the modal assurance criterion (MAC) function, as shown in Eq. (23).

$$
\operatorname{MAC}\left(\left\{\phi_{o}\right\},\left\{\phi_{s}\right\}\right)=\frac{\left|\left\{\phi_{o}\right\}^{H}\left\{\phi_{s}\right\}\right|^{2}}{\left(\left\{\phi_{o}\right\}^{H}\left\{\phi_{o}\right\}\right)\left(\left\{\phi_{s}\right\}^{H}\left\{\phi_{s}\right\}\right)}
$$

, where $\phi_{o}$ is the UMM shape vector of oblique MA, and $\phi_{s}$ is the UMM shape vector of superposition-normal MA. $\bullet^{H}$ denotes the Hermitian transpose, i.e. complex conjugate transpose function. MAC value is calculated between 0 and 1 . MAC value of 1 indicates a 
perfect correlation between two mode shapes, while the MAC value of 0 indicates uncorrelated mode shape. In practice, MAC value greater than 0.9 is commonly recognized as similar mode shapes or consistent correspondence [32].

\section{Results and Discussion}

\subsection{Comparison of FRF Results between Oblique and Tri-Axial Normal Impact Testing}

Measured oblique FRFs of 57 sets and superposition-normal FRFs of 57 sets are overlaid in Figs. 7 and 8 respectively. Both figures show three global peaks within the first $50 \mathrm{~Hz}$. Each global peak indicates the vibration modes of the T-shaped structure. From the visual comparison, both FRFs show good agreement of FRF pattern from 0 to $50 \mathrm{~Hz}$. The location of the peak, as well as the peaks' magnitude are close and similar. The discrepancy between the maximum peak magnitude of oblique and superposition-normal FRFs are shown in Table 1. The percentage of differences between the magnitudes of the peaks are less than $3 \%$, which indicates good agreement between both FRFs.

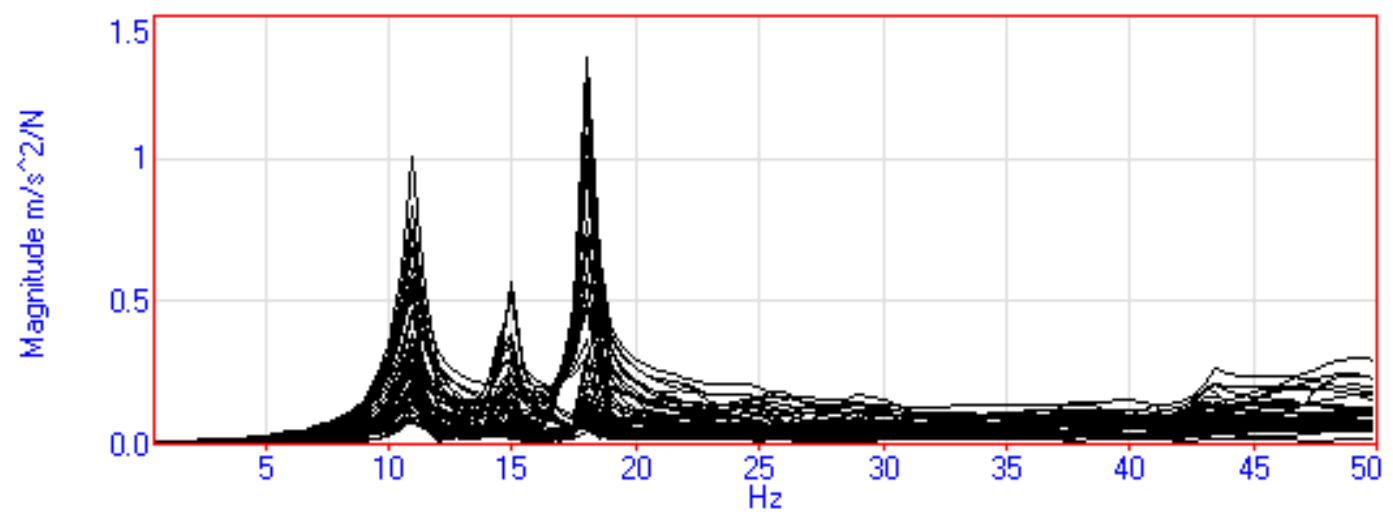

Fig. 7. Overlaid of all oblique FRFs result from oblique impact testing. 


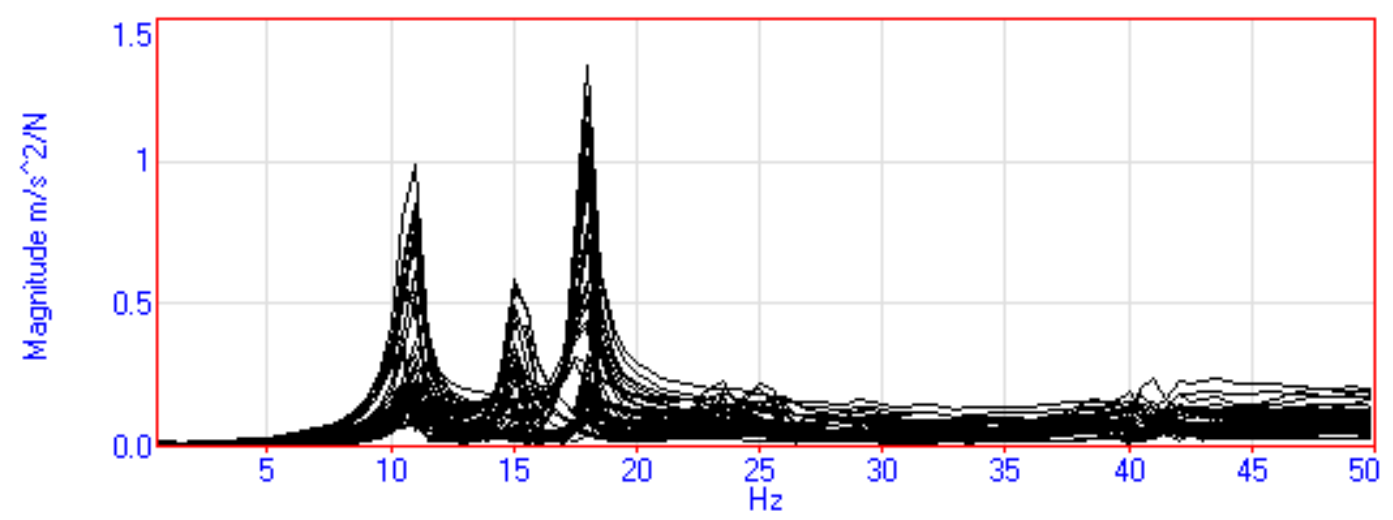

Fig. 8. Overlaid of all superposition-normal FRFs result from tri-axial normal impact testing.

\section{Table 1}

Comparison of maximum peak magnitude of superposition-normal and oblique FRFs.

\begin{tabular}{cccc}
\hline Peak & $\begin{array}{r}\text { Oblique FRF, } \\
\mathrm{ms}^{-2} / \mathrm{N}\end{array}$ & $\begin{array}{c}\text { Superposition-Normal FRF, } \\
\mathrm{ms}^{-2} / \mathrm{N}\end{array}$ & $\%$ \\
\hline 1 & 1.0065 & 0.9894 & 1.73 \\
2 & 0.5683 & 0.5865 & 3.00 \\
3 & 1.3644 & 1.3416 & 1.70 \\
\hline
\end{tabular}

Furthermore, oblique and superposition-normal FRFs are averaged respectively and the results are plotted in Fig. 9. Then, the correlation value is calculated to examine the closeness of oblique FRF to superposition-normal FRF. As a result, a correlation value of 0.92 is obtained, which indicates that the oblique FRF has good agreement with the superposition-normal FRF. 


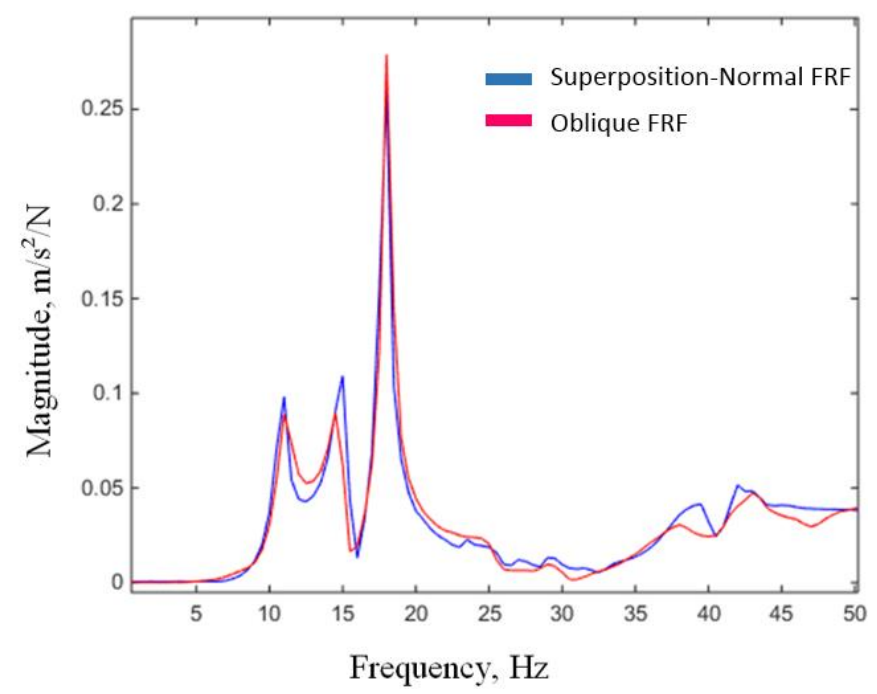

Fig. 9. Mean superposition-normal FRF versus mean oblique FRF.

Side by side comparison of the oblique and superposition-normal FRFs in various directions of the response DOF is shown in Fig. 10. Closer examination of it shows great agreement in terms of the peak magnitude and peak location as well as the FRF pattern for various directions of FRF's response DOF. The result shows that the oblique FRF matches well with the superposition-normal FRF, and thus the 'concurrent forces effect' of the oblique FRF is validated. With that, significant improvement is successfully demonstrated in this study, against the oblique impact testing result reported in the previous studies [20,21]. Those studies did not consider the theoretical linkage of the oblique FRF with normal FRF, therefore these studies could not explain well the variations in the obtained FRF pattern and mode shape, as compared to the normal FRF result from the uni-axial impact testing. The main reason for the variation is discovered in this study, where a single column/row of normal FRF obtained from the uni-axial normal impact testing is only a part of the oblique FRF. Considering the 'concurrent forces effect' of oblique FRF that excites the structure in all principal directions, oblique FRF is validated to closely approximate the superposition-normal FRF, which is formed from the superposition of all columns/rows of normal FRF obtained from the trial-axial normal impact testing, as follows Eq. (20). 
(a)

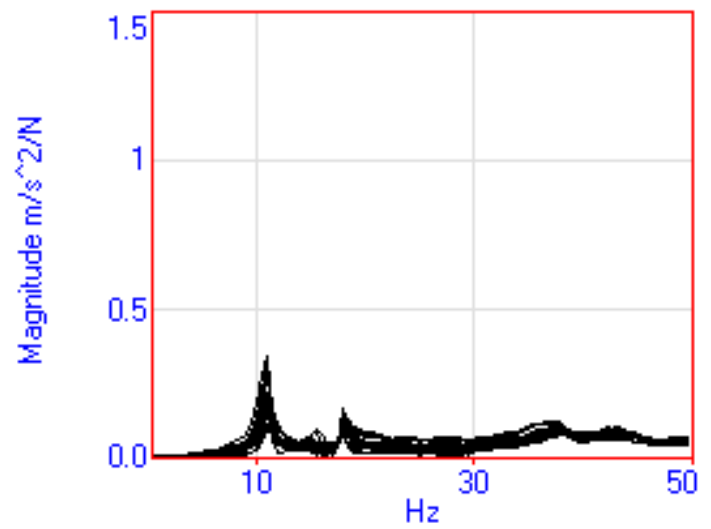

(b)

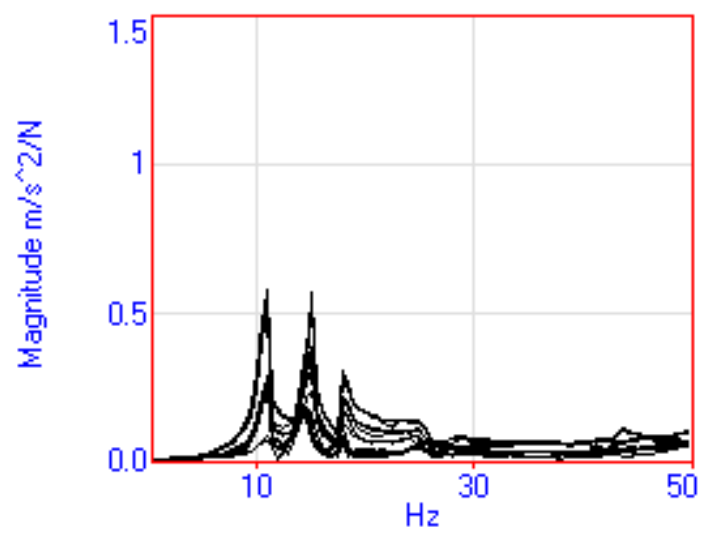

(c)

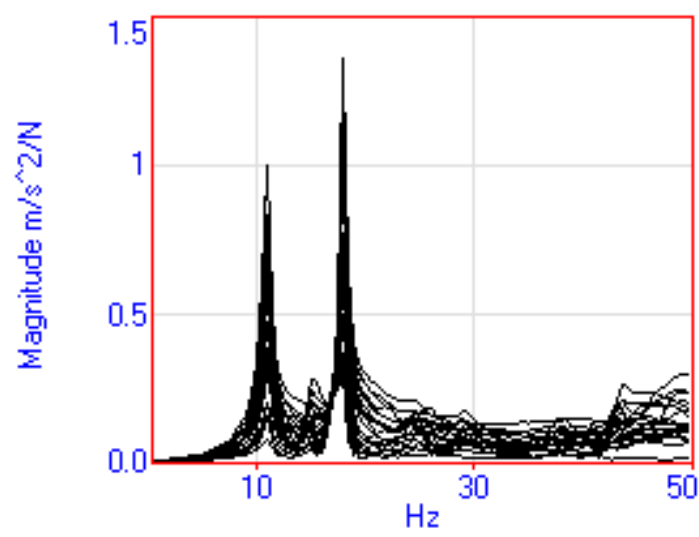

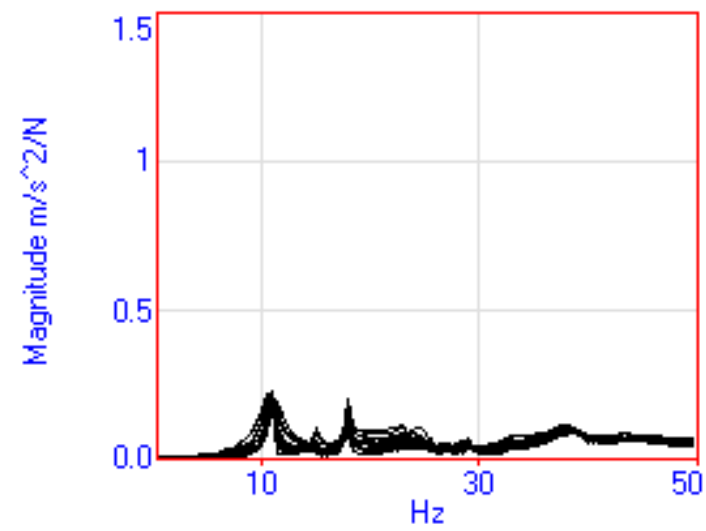
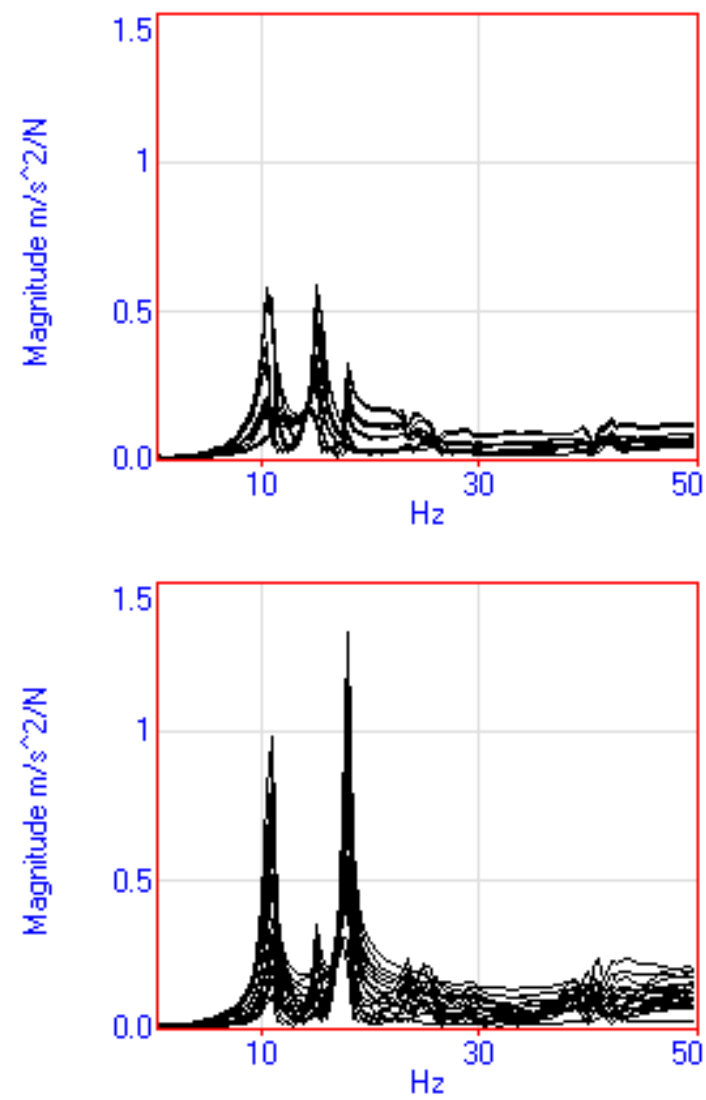

Fig. 10. Side by side comparison between oblique (left) and superposition-normal (right) FRFs due to response DOF in (a) $x$-, (b) $y$-, and (c) $z$-directions.

\subsection{Comparison of Modal Parameter Results between Oblique and Tri-Axial Normal Impact}

\section{Testing}

To examine the accuracy of the proposed oblique impact testing in determining the structural dynamic characteristics, benchmarking with the modal parameter result from the conventional 
tri-axial normal impact testing is necessary. This can be done by taking the oblique and superposition-normal FRFs including its driving point measurement into a modal curve fitting process. A total of 3 vibration modes are obtained from the curve fitting result, as they manifest predominantly vertical and horizontal motions. Hence, a comparison of various modal parameters is performed in terms of the natural frequency result (Table 2), modal damping ratio result (Table 3) and mode shape result (Figs. 11 - 13 and Table 4).

Table 2 shows that the natural frequencies obtained from the oblique impact testing have an accuracy range within $98.34 \%-98.83 \%$, as compared to the result of tri-axial normal impact testing. Since the obtained percentage of discrepancy is less than $2 \%$, oblique impact testing can estimate the natural frequencies accurately. Table 3 shows the modal damping ratios obtained from both tests. Note that the absolute error is being adopted in comparing the results instead of using the percentage of discrepancy, as follows the previous literature [33]. This is because the examination of the damping ratio in the percentage of discrepancy could easily incur the exaggeration effect that may cause wrong analysis. The obtained absolute error has range within $0.07 \%-0.99 \%$. Usually, accurate modal damping ratio estimation is difficult in nature and it is often expected to be less certain. This is because the measurement of this parameter is very sensitive to the small change of the boundary and ambient conditions, as reported on the finding of Luis et al. [34] and Philip et al. [35]. In this study, small inconsistency of impact direction, small deviation of the test rig's position, and small variation of the loading position for various loading conditions, such as oblique and normal impacts, may lead to notable variation in the modal damping ratio estimation. Despite the estimation variation, since the modal damping ratios obtained from both tests are relatively small for structural applications (i.e. lightly damped structure) as they are less than 5\% [36]. The proposed testing 
can estimate the lightly damped characteristic of the test rig successfully. Thus, the estimation of the modal damping ratio is satisfactory.

Table 2

Natural frequencies obtained from oblique and tri-axial normal impact testing.

\begin{tabular}{cccc}
\hline Mode & $\begin{array}{c}\text { Natural Frequency from } \\
\text { Oblique Impact Testing }\end{array}$ & $\begin{array}{c}\text { Natural frequency from Tri- } \\
\text { Axial Normal Impact Testing }\end{array}$ & $\begin{array}{c}\text { Percentage of } \\
\text { Discrepancy }\end{array}$ \\
\hline $\mathbf{1}$ & 11.00 & $\mathbf{( H z )}$ & $(\boldsymbol{\%})$ \\
\hline $\mathbf{2}$ & 14.81 & 10.83 & 1.57 \\
\hline $\mathbf{3}$ & 18.09 & 15.06 & 1.66 \\
\hline
\end{tabular}

Table 3

Modal damping ratio obtained from oblique and tri-axial normal impact testing.

\begin{tabular}{|c|c|c|c|}
\hline Mode & $\begin{array}{l}\text { Modal Damping Ratio } \\
\text { from Oblique Impact }\end{array}$ & $\begin{array}{l}\text { Modal Damping Ratio from } \\
\text { Tri-Axial Normal Impact }\end{array}$ & $\begin{array}{c}\text { Absolute Error } \\
\qquad(\%)\end{array}$ \\
\hline & $\begin{array}{l}\text { Testing } \\
(\%)\end{array}$ & $\begin{array}{l}\text { Testing } \\
(\%)\end{array}$ & \\
\hline 1 & 3.47 & 2.48 & 0.99 \\
\hline 2 & 1.99 & 2.50 & 0.51 \\
\hline 3 & 1.26 & 1.33 & 0.07 \\
\hline
\end{tabular}

The third dynamic characteristic is the UMM mode shape. Both UMM mode shapes obtained from the oblique and normal impact tests are compared side by side in various views, as shown in Figs. 11 - 13. For the vibration mode \#1, the mode shape is dominated by the swaying and 
pitching motions, which is clearly shown in Figs. 11(a) and 11(d). Mode \#2 is dominated by swaying and rolling motions, as shown in Figs. 12(a) and 12(c). Figs. 13 shows that mode \#3 is dominated by the heaving motion. From the visual comparison, it shows that both mode shapes agree well with each other for all examined vibration modes. Furthermore, mode shapes are compared quantitatively using MAC in Table 4. It shows that all modes have MAC values greater than 0.9 at the diagonal term, which indicates that all mode shapes obtained from the proposed oblique impact testing are very similar to the UMM mode shapes obtained from the conventional tri-axial normal impact testing result. A non-correlated relationship is found at the off-diagonal term $(\mathrm{MAC}<0.1)$ which indicates that the mode shapes are unrelated or independent between two mode shapes at different modes. Besides, the off-diagonal term with near-zero value indicates that it is free of spatial aliasing issue [32]. In overall, the oblique impact testing successfully estimates the UMM mode shape with high accuracy.

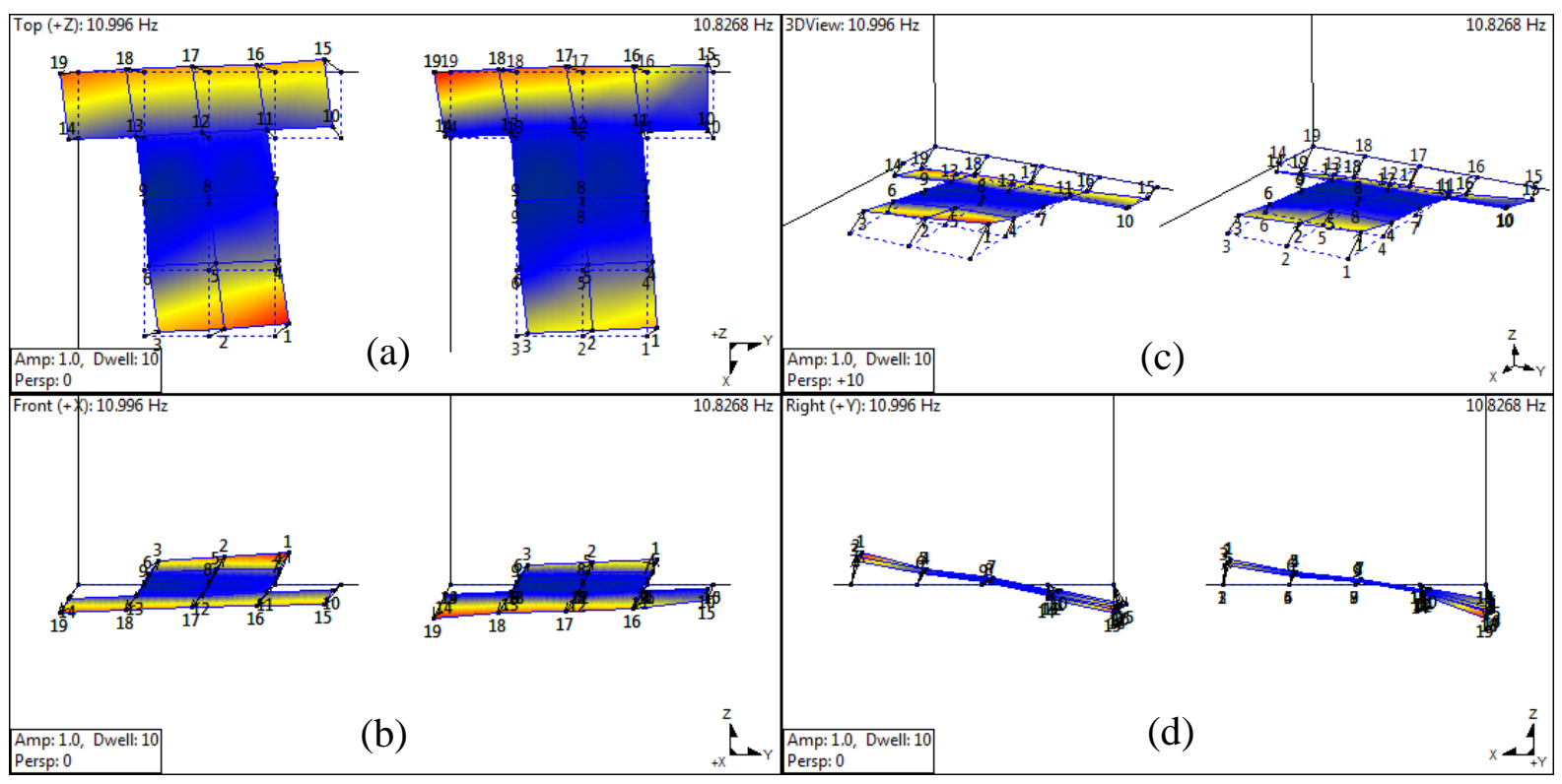

Fig. 11. Side by side comparison between UMM mode shape \#1 obtained from oblique (left) and normal (right) impact testing: (a) top, (b) front, (c) isometric, and (d) right views. 


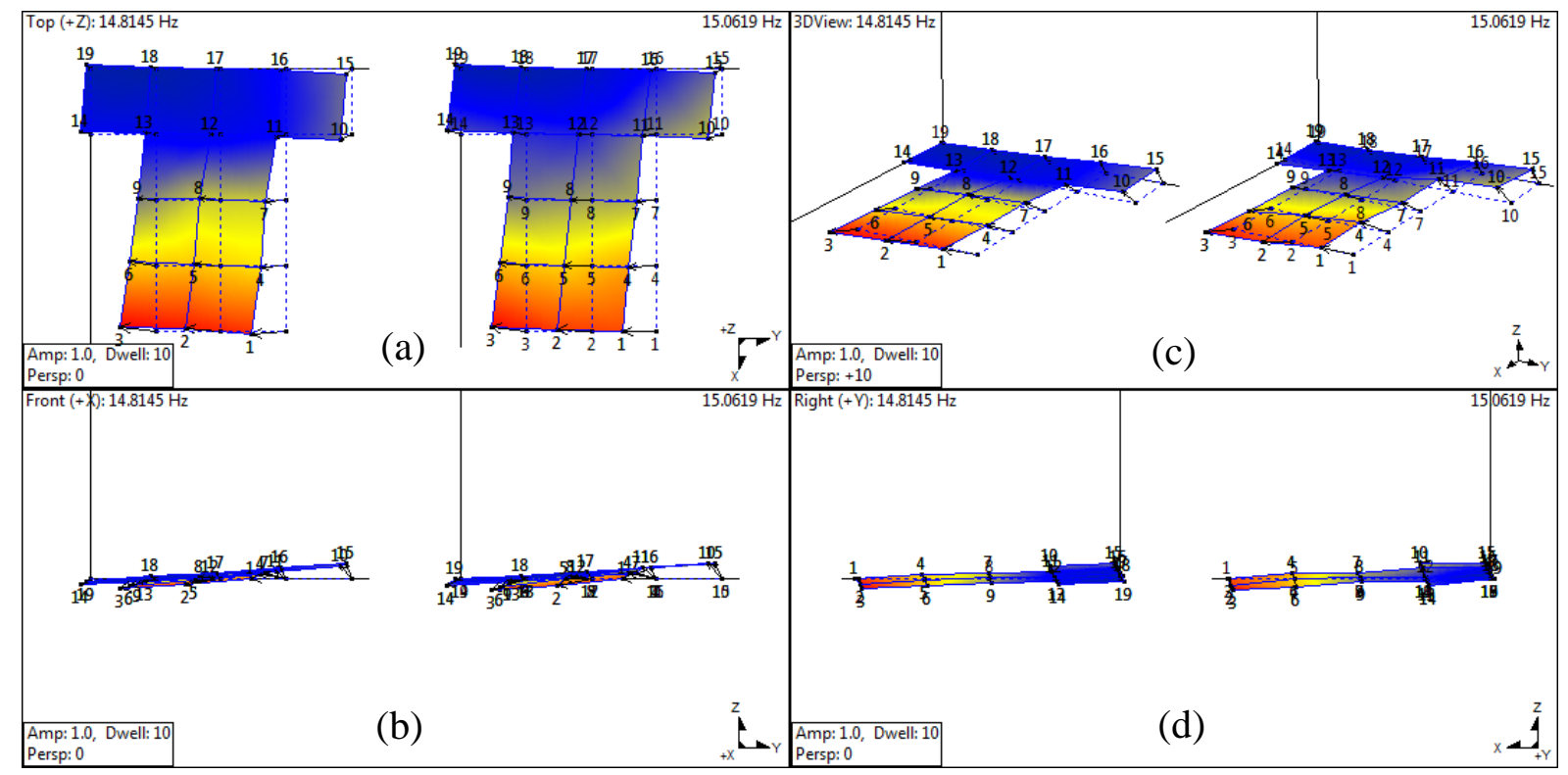

Fig. 12. Side by side comparison between UMM mode shape \#2 obtained from oblique (left) and normal (right) impact testing: (a) top, (b) front, (c) isometric, and (d) right views.

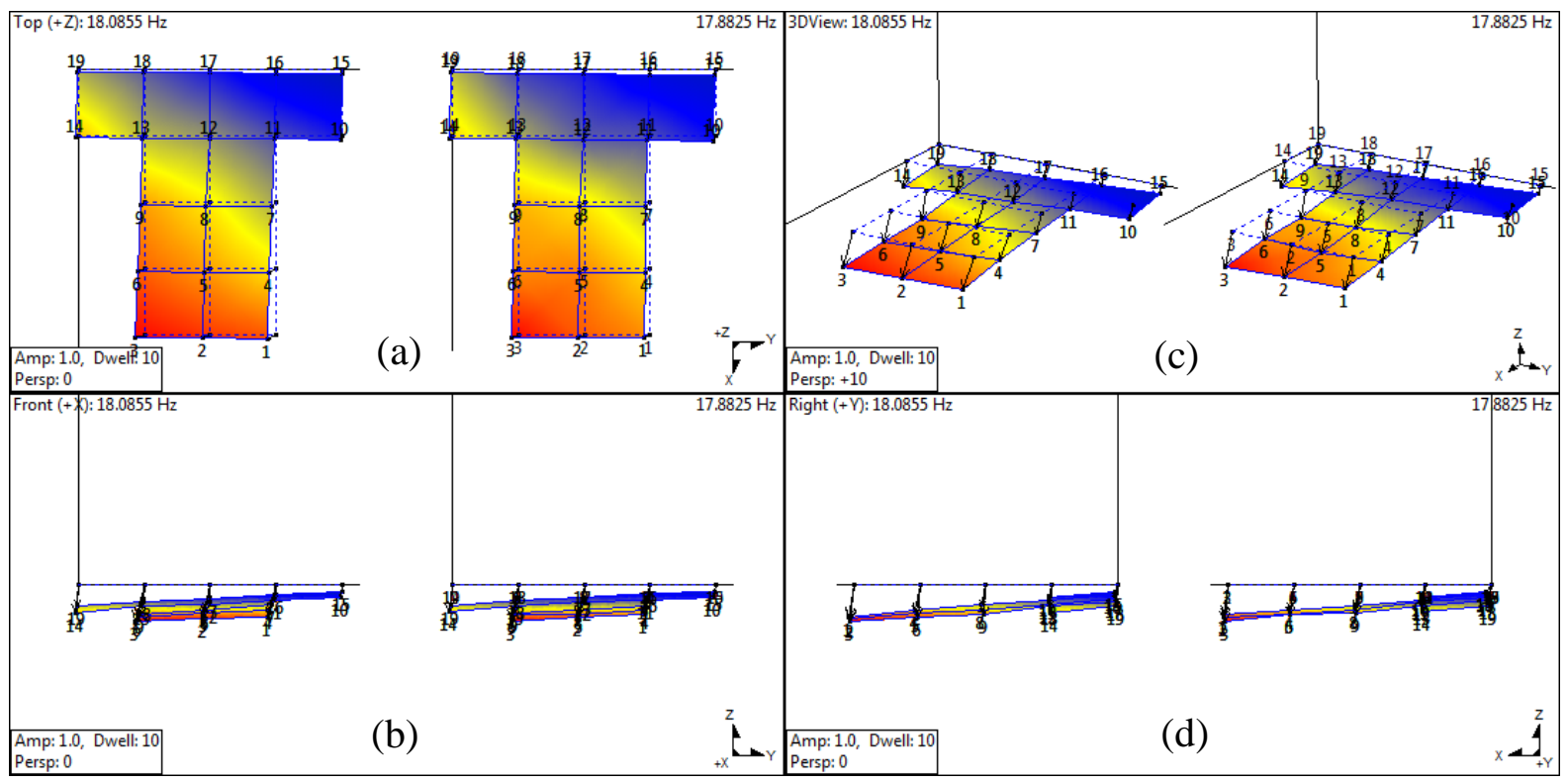

Fig. 13. Side by side comparison between UMM mode shape \#3 obtained from oblique (left) and normal (right) impact testing: (a) top, (b) front, (c) isometric, and (d) right views. 


\section{Table 4}

MAC result between UMM mode shapes obtained from the oblique and tri-axial normal impact testing.

\begin{tabular}{|c|c|c|c|c|c|}
\hline Normal & & & Mode Shape & Mode Shape & Mode Shape \\
\hline Impact & & & $\# 1$ & $\# 2$ & $\# \mathbf{3}$ \\
\hline Case & $\begin{array}{c}\text { Frequency } \\
(\mathbf{H z})\end{array}$ & & 10.83 & 15.06 & 17.88 \\
\hline $\begin{array}{l}\text { Impact } \\
\text { Case }\end{array}$ & & $\begin{array}{c}\text { Damping } \\
(\%)\end{array}$ & 2.48 & 2.50 & 1.33 \\
\hline Mode Shape \#1 & 11.00 & 3.47 & 0.903 & 0.046 & 0.054 \\
\hline Mode Shape \#2 & 14.81 & 1.99 & 0.063 & 0.949 & 0.033 \\
\hline Mode Shape \#3 & 18.09 & 1.26 & 0.036 & 0.040 & 0.972 \\
\hline
\end{tabular}

\section{Conclusion}

In this study, the reliability of the fixture-free oblique impact testing has been examined. Additional driving point FRF measurement in the oblique direction has been successfully obtained by using the proposed vector projection method. This method eliminates the need of a special fixture for the placement of an oblique-oriented accelerometer/impedance head which greatly increases the practicality of oblique impact testing. The theoretical relationship between the oblique and conventional tri-axial normal impact testing is successfully developed in this study. Validation of the proposed oblique impact testing is successfully performed through the comparison of the measured FRF and curve-fitted modal parameters, with the results of triaxial normal impact testing. Experimental results show that the oblique FRF matches the superposition-normal FRF well, with a high correlation value of 0.92. Furthermore, the percentage of discrepancies less than $2 \%$ is obtained for the natural frequencies estimation, and 
absolute errors of less than $1 \%$ are obtained for the modal damping ratios estimation. Driving point FRF is successfully used to scale the residue mode shape, and the obtained UMM mode shapes have great accuracy, with MAC value greater than 0.903 for all the examined modes. To a great degree, oblique impact testing is validated to be an alternative and reliable solution for performing the experimental modal analysis, rather than performing a time-consuming triaxial normal impact testing.

\section{Acknowledgement}

The authors wish to acknowledge the financial support given by the Fundamental Research

Grant Scheme (FP057-2015A) and the advice given by the Advanced Shock and Vibration Research (ASVR) Group of University of Malaya and other project collaborators.

\section{Reference}

1. Herman, V.d.A., et al., Structural Design Changes as a Solution to a Resonance Fatigue Problem of a Sports Car. 1993, SAE Technical Paper.

2. $\quad$ Eiras, J.N., et al., Experimental modal analysis and finite element model updating for structural health monitoring of reinforced concrete radioactive waste packages. Construction and Building Materials, 2018. 180: p. 531-543.

3. Roy, H. and S. Chandraker, Dynamic study of viscoelastic rotor: Modal analysis of higher order model considering various asymmetries. Mechanism and Machine Theory, 2017. 109: p. 65-77.

4. $\quad \mathrm{Wu}, \mathrm{Q}$., et al., Crack diagnosis method for a cantilevered beam structure based on modal parameters. Measurement Science and Technology, 2019. 31(3): p. 035001.

5. Fan, W. and P. Qiao, Vibration-based damage identification methods: a review and comparative study. Structural health monitoring, 2011. 10(1): p. 83-111.

6. Khoo, S.Y., et al., Impact force identification with pseudo-inverse method on a lightweight structure for under-determined, even-determined and over-determined cases. International Journal of Impact Engineering, 2014. 63: p. 52-62.

7. Kong, K.K., et al., Identification of material properties of orthotropic composite plate using hybrid non-destructive evaluation approach. Materials Research Innovations, 2014. 18: p. 423-428.

8. Kouroussis, G., L.B. Fekih, and T. Descamps, Assessment of timber element mechanical properties using experimental modal analysis. Construction and Building Materials, 2017. 134: p. 254-261.

9. $\mathrm{Li}, \mathrm{H}$., et al., An iterative method for identification of temperature and amplitude dependent material parameters of fiber-reinforced polymer composites. International Journal of Mechanical Sciences, 2020. 184: p. 105818. 
10. Ewins, D., Basics and state-of-the-art of modal testing. Sadhana, 2000. 25(3): p. 207220.

11. Keyhani, A. and S. Mohammadi, Structural modal parameter identification using local mean decomposition. Measurement Science and Technology, 2018. 29(2): p. 025003.

12. Yanez-Borjas, J.J., et al., Nonlinear mode decomposition-based methodology for modal parameters identification of civil structures using ambient vibrations. Measurement Science and Technology, 2019. 31(1): p. 015007.

13. XiaoLi, Z., et al., An inverse decaying frequency modulation EMD method for closely spaced modal parameter identification in high precision with Laplace wavelet correlation filtering. Measurement Science and Technology, 2020.

14. Brown, D.L., R.J. Allemang, and A.W. Phillips, Forty years of use and abuse of impact testing: a practical guide to making good FRF measurements, in Experimental Techniques, Rotating Machinery, and Acoustics, Volume 8. 2015, Springer. p. 221-241.

15. Ewins, D.J., Modal Testing: Theory, Practice, and Application. 2000, England: Research Studies Press LTD.

16. Schwarz, B.J. and M.H. Richardson, Experimental modal analysis. CSI Reliability week, 1999.

17. Daborn, P.M., P. Ind, and D. Ewins, Enhanced ground-based vibration testing for aerodynamic environments. Mechanical Systems and Signal Processing, 2014. 49(1-2): p. 165-180.

18. Aykan, M. and M. Celik, Vibration fatigue analysis and multi-axial effect in testing of aerospace structures. Mechanical Systems and Signal Processing, 2009. 23(3): p. 897907.

19. Okyay, A., K. Erkorkmaz, and M. Khamesee, Modal Analysis, Metrology, and Error Budgeting of a Precision Motion Stage. Journal of Manufacturing and Materials Processing, 2018. 2(1): p. 8.

20. Khoo, S.Y., et al., Time effective structural frequency response testing with oblique impact, in 20th International Conference on Acoustics, Vibration and Noise Control Engineering (ICAVNCE). 2018: Venice, Italy.

21. Døssing, O., Improvement to monoreference modal data by adding an oblique degree of freedom for the reference, in Proceedings of the International Modal Analysis Conference IMAC IV. 1986: Los Angeles Airport Marriott Hotel, Los Angeles, CA. p. pp. 23-42.

22. Herlufsen, H., Modal analysis using multi-reference and multiple-input multiple-output techniques. Brüel \& Kjær, Application Note, 2004: p. 16.

23. Avitabile, P., MODAL SPACE: If I run a shaker test with the input oblique to the global coordinate system, how do I decompose the force into the specific components in each direction? Experimental Techniques, 2009. 33(5): p. 11-12.

24. Warren, C., et al., Comparison of FRF measurements and mode shapes determined using optically image based, laser, and accelerometer measurements. Mechanical Systems and Signal Processing, 2011. 25(6): p. 2191-2202.

25. Baqersad, J., et al., Comparison of modal parameters extracted using MIMO, SIMO, and impact hammer tests on a three-bladed wind turbine, in Topics in Modal Analysis II, Volume 8. 2014, Springer. p. 185-197.

26. Lauffer, J.P., T.G. Carne, and T.D. Ashwill, Modal testing in the design evaluation of wind turbines. 1988: Sandia National Laboratories.

27. Richardson, M. and B. Schwarz, Modal parameter estimation from operating data. Sound and Vibration, 2003. 37(1): p. 28-39.

28. Richardson, M. and C. Jamestown, Modal mass, stiffness and damping. Vibrant Technology, Inc., Jamestown, CA, 2000: p. 1-5. 
29. Richardson, M.H. and C. Jamestown, Modal mass, stiffness and damping. Vibrant Technology, Inc., Jamestown, CA, 2000: p. 1-5.

30. ISO 7626-1:2011 Vibration and shock - Experimental determination of mechanical mobility, in Part 1: Basic terms and definitions, and transducer specifications. 2011.

31. ISO 7626-5:1994 Vibration and shock - Experimental determination of mechanical mobility, in Part 5: Measurements using impact excitation wth an exciter which is not attached to the structure. 1994.

32. Pastor, M., M. Binda, and T. Harčarik, Modal Assurance Criterion. Procedia Engineering, 2012. 48(0): p. 543-548.

33. Mbarek, A., et al., Comparison of experimental and operational modal analysis on a back to back planetary gear. Mechanism and Machine Theory, 2018. 124: p. 226-247.

34. Felipe-Sesé, L., et al., FP+DIC for low-cost 3D full-field experimental modal analysis in industrial components. Mechanical Systems and Signal Processing, 2019. 128: p. 329-339.

35. Reu, P.L., D.P. Rohe, and L.D. Jacobs, Comparison of DIC and LDV for practical vibration and modal measurements. Mechanical Systems and Signal Processing, 2017. 86: p. 2-16.

36. Giurgiutiu, V., Structural health monitoring: with piezoelectric wafer active sensors. 2007: Elsevier. 